Pacific Journal of Mathematic 


\section{COVERING RELATIONS AMONG LATTICE VARIETIES}

\section{DANG X. HoNG}

o. Introduction. It is shown in this paper that the equational class generated by the family of all projective planes can be characterized by a finite set of lattice identities. The methods developed here may provide a framework to attack similar problems and a useful medium for studying modular lattices in general.

By a variety, or equational class, of lattices we mean the class of all lattices satisfying a given set of lattice identities. A lattice variety is finitely based if it can be defined by a finite set of identities. Let $\Lambda$ be the lattice of all lattice varieties. A systematic study of the lattice $\Lambda$ dates back seven or eight years ago. Most recent results in this field, including ours here, are stimulated by an important discovery of Bjarni Jónsson in [7], Corollary 3.2. (See Baker [1], [2], Grätzer [4], Hong [5], Jónsson [7], [8], McKenzie [9], [10], Wille [11].) Our study here continues the works of Grätzer in [4] and of Jónsson in [8], where the latter completed an unfinished result of the former and in particular proved that the variety generated by all projective lines is finitely based.

The rest of the paper is divided into four sections. In $\S 1$ we state our main theorem and its applications but postpone the proofs until $\S 4$. In $\S 2$ we discuss the main methods employed here: the method of strong covering, and the notions of normality and strong normality of sequences of transposes. In case the family of all varieties that strongly covers a given variety is finite, then the variety is finitely based. The notions of normality and strong normality, due to Grätzer and Jónsson respectively, are developed rather completely in Theorem 3.1. We hope that this theorem will have some applications elsewhere. Section 4 gives details of the proof of the main lemma stated in Section 1.

In the sequel, almost every theorem and lemma has its dual, even though we rarely make explicit mention of this fact. Also, the notation $L$ denotes a fixed modular lattice.

We wish to express our sincere gratitude to Professor Bjarni Jónsson for his helpful suggestions in the ideas as well as the presentation of this paper. We also wish to thank the referee for his detailed suggestions.

1. The main theorem and its applications. For any family $K$ of lattices, let $\boldsymbol{S}(\boldsymbol{K}), \boldsymbol{H}(\boldsymbol{K}), \boldsymbol{P}_{\boldsymbol{u}}(\boldsymbol{K})$ denote respectively the families of sublattices, of homomorphic images and of ultraproducts of members 
of $\boldsymbol{K}$. The important discovery of Jonsson, mentioned in the introduction, asserts that every subdirectly irreducible member of the variety generated by a family $\boldsymbol{K}$ of lattices belongs to $\boldsymbol{H S P}_{\boldsymbol{u}}(\boldsymbol{K})$. Let $\boldsymbol{A}_{1}, \boldsymbol{A}_{2}$, $A_{3}, M_{3 k}$, where $k$ is a positive integer, denote the lattices in Fig. 1.
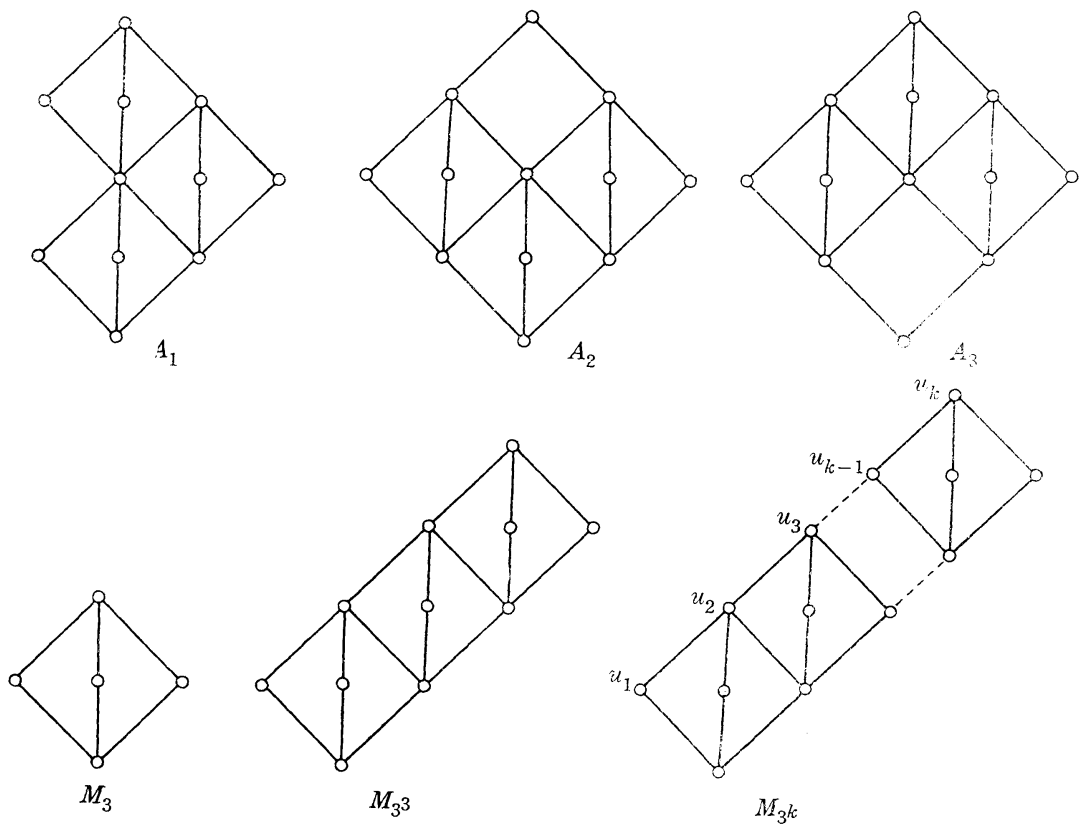

FIGURE 1

We first state the main lemma, the proof of which is postponed until $\S 4$.

1.1. MaIN Lemma. Let $L$ be a subdirectly irreducible modular lattice and

$$
A_{1}, A_{2}, A_{3}, M_{3 n} \notin \boldsymbol{H} \boldsymbol{S}(L),
$$

where $n$ is a positive integer. Then $\operatorname{dim}(L) \leqq n$.

The main application of this lemma is to prove the principal result of the paper by the use of the notion of strong covering. Consider a variety $\mathscr{W}$ and let $C(\mathscr{U})$ be the family of all varieties that cover $\mathscr{W}$. A family of varieties $\left\{\mathscr{V}_{i} \mid i \in I\right\}$ is said to strongly cover $\mathscr{W}$ if every $\mathscr{V}_{i}, i \in I$, covers $\mathscr{W}$ and any variety that properly contains $\mathscr{W}$ contains a variety $\mathscr{Y}_{i}$ for some $i \in I$. Then for each $i \in I$ there is a lattice identity $\theta_{i}$ that holds in $\mathscr{W}$ but not in $\mathscr{V}_{i}$. It is easy to see that modulo the lattice axioms, $\mathscr{V}$ is the variety defined by $\left\{\theta_{i} \mid i \in I\right\}$. Thus if $I$ is finite, then $\mathscr{\mathscr { P }}$ is finitely based. (It is still an open question whether $C(\mathscr{U})$ always strongly covers $\mathscr{W}$ for any variety $\mathscr{W}$.) Now combining the Main Lemma, the above 
mentioned result of Jonsson and the characterization of all modular lattices of dimension $\leqq 3$ in [6], one can easily prove the following theorem and its corollaries.

1.2. MAIN THEOREM. Let $\mathscr{M}_{\infty}^{3}$ be the variety generated by the family of all projective planes. The five varieties generated by $\mathscr{M}_{\infty}^{3}$ and each member of the family

$$
\left\{A_{1} A_{2}, A_{3}, M_{3}, N_{5}\right\} \text {, }
$$

where $N_{5}$ is the five-element nonmodular lattice, form a strong cover for $\mathscr{L}_{\infty}^{3}$.

COROLlaRY 1. The variety $\mathscr{L}_{\infty}^{3}$ is finitely-based.

CoRollary 2. Let $\mathscr{W}$ be the variety generated by any finite projective plane. Then $\boldsymbol{C}(\mathscr{W})$ is finite, can be effectively found and strongly covers $\mathscr{W}$. Consequently, $\mathscr{W}$ is finitely based.

REMARK. With some details added, one can show that the conclusions of Corollary 2 still hold when $\mathscr{W}$ is the variety generated by $\boldsymbol{P} \cup \boldsymbol{Q}, \mathscr{N}_{\infty}^{2} \cup \boldsymbol{P} \cup \boldsymbol{Q}$, or $\mathscr{N}_{\infty}^{2} \cup \boldsymbol{P} \cup \boldsymbol{Q}$, where $\mathscr{H}_{\infty}^{2}$ is the variety generated by all projective lines, and

$\boldsymbol{P}$ is a finite family of finite modular lattices of dimension less than or equal to 3 ,

$\boldsymbol{Q}$ is a finite family of finite modular lattices of the form $M_{\left[n_{1}, n_{2} \cdots, n_{k}\right]} \cdot$

For the definition of $M_{\left[n_{1}, n_{2}, \cdots, n_{k}\right]}$ and the details of the proof, the reader is referred to the author's thesis [5].

Concluding this section, we state some conjectures for which we hope the methods here may be helpful.

Conjecture 1. The variety generated by a finite modular lattice is strongly covered by a finite number of varieties.

Conjecture 2. For each positive integer $i$, the variety $\mathscr{M}_{\infty}^{i}$ generated by the family of all modular lattices of dimension $\leqq i$ is strongly covered by a finite number of varieties.

\section{Methods.}

A. Diamond, transpositions and translations.

1. Diamond. By a diamond we mean a five-termed sequence $[u \geqq x, y, z \geqq v]$ of elements of $L$ whose terms are all equal (in which case the diamond is said to be degenerate) or else form a non-distributive lattice $M_{3}$ in Fig. 1 . The intervals $[x, u],[y, u],[z, u]$ are called 
the first, second and third upper edges, and $[v, x],[v, y],[v, z]$ the first, second and third lower edges. For any two members $t, t^{\prime}$ of $\{x, y, z\}$, the intervals $[t, u]$ and $\left[v, t^{\prime}\right]$ are said to be adjacent if $t=t^{\prime}$; otherwise they are said to be opposite. In case there is no ambiguity we can identify a diamond with the sublattice whose elements are its terms; the order of the vertices is then irrelevant.

2. Transpositions. Translations. It is well known that an interval $[a, b]$ is said to transpose down onto an interval $[c, d]$, or $[c$, $d]$ up onto $[a, b]$, if $a+d=b$ and $a d=c$. We write then

$$
[a, b] \succ[c, d], \text { or }[c, d] \succ[a, b] \text {. }
$$

We know that the mappings $\phi: r \rightarrow r d$ for every $r$ in $[a, b]$ and $\psi$ : $s \rightarrow s+a$ for every $s$ in $[c, d]$ are isomorphisms of $[a, b]$ onto $[c, d]$ and $[c, d]$ onto $[a, b]$ respectively. They are called the transposition between these two intervals. We now come to the definitions of transpositions and translations between two diamonds, say $D_{i}=\left[u_{i} \geqq x_{i}, y_{i}, z_{i} \geqq v_{i}\right]$, $i=1,2$. We say that

(i) $D_{1}$ transposes down onto $D_{2}$, or $D_{2}$ transposes up onto $D_{1}$, in symbols

$$
D_{1} \underset{(1)}{\longrightarrow} D_{2} \text {, or } D_{2} \underset{(1)}{\longrightarrow} D_{1}
$$

if $\left[v_{1}, u_{1}\right]$ transposes down onto $\left[v_{2}, u_{2}\right]$ and in this transposition the vertices $x_{1}, y_{1}, z_{1}$ are mapped into the corresponding vertices $x_{2}, y_{2}, z_{2}$.

(ii) $D_{1}$ translates down onto $D_{2}$, or $D_{2}$ translates up onto $D_{1}$, if a lower edge of $D_{1}$ transposes down onto an upper edge of $D_{2}$. If specifically $\left[v_{1}, z_{1}\right]$ transposes down onto $\left[x_{2}, u_{2}\right]$, then we write

$$
D_{1} \underset{(2)}{>} D_{2} \text {. }
$$

Also, if $D_{1}$ translates up onto $D_{2}$ by $\left[z_{1}, u_{1}\right]$ transposing up onto $\left[v_{2}, x_{2}\right]$, then we write

$$
D_{1} \underset{\text { (2) }}{\longrightarrow} D_{2} \text {. }
$$

Note that $D_{1} \nearrow_{(2)} D_{2}$ does not imply $D_{2} \searrow_{(2)} D_{1}$ •

The following lemma will be frequently used subsequently.

3. Transposition Lemma. Suppose $[a, b]$ and $[c, d]$ are two intervals in $L$.

(i) If $D=[u \geqq x, y, z \geqq v]$ is a diamond such that

$$
[z, u] \supset[c, d] \succ[a, b] \text { and } a+v=c,
$$

then we have a diamond [ $b u \geqq b x, b y, b z \geqq b v]$, in symbols $b D$, satisfying 
the condition $D \underset{(1)}{\searrow} b D$ and $[b z, b u] \nearrow[a, b]$.

(ii) If $[v, u]^{(1)}$ is an interval such that

$$
[v, u]>[c, d]>[a, b] \text { and } a+v=c,
$$

then $[v, u] \searrow[b v, b u] \nearrow[a, b]$.

Proof. Suppose (1) holds. Clearly $b+v=d$, hence $[v, d]$ transposes down onto $[b v, b]$. Under this transposition, $b D,[b z, b u]$ and $[a, b]$ are respectively the images of $D,[z, u]$ and $[c, d]$. Therefore $b D$ is a diamond, $D$ transposes down onto $z D$, and $[b z, b u]$ transposes up onto $[a, b]$.

The proof of (ii) is equally trivial.

REMARK. Observe that the part (i) of the lemma also holds when $[z, u]$ is replaced by any upper edge of $D$. In later applications, we will use this observation without mentioning it.

4. String of diamonds. Given a sequence of $k$ diamonds, $D_{1}, D_{2}$, $D_{3}, \cdots, D_{i}, \cdots, D_{k}$ such that for $1<i<k, D_{i}$ translates up onto $D_{i+1}$ and down onto $D_{i-1}$ by two opposite edges, the sublattice formed by these diamonds is called a string of $k$ diamonds, denoted in each occurrence by $M_{3^{k}}^{+}$. Thus, $M_{3^{k}}$ is also a string of $k$ diamonds.

B. Projectivities and projective distance. Two intervals $[a, b]$ and $[c, d]$ are said to be connected by the sequence of transposes $\left[a_{k}, b_{k}\right]$, $k=0,1,2, \cdots, n$, if $\left[a_{0}, b_{0}\right]=[a, b]$ and $\left[a_{n}, b_{n}\right]=[c, d]$, and for $k=0$, $1,2 \cdots, n-1$, the $k$ th term transposes alternately up and down onto the next. Two intervals are said to be projective in $n$ steps if they are connected by an $n+1$-termed sequence of transposes. For any two intervals $[a, b]$ and $[c, d]$, let $P([a, b],[c, d])$ be the smallest nonnegative integer with the property that there exist nontrivial subintervals $[\bar{a}, \bar{b}]$ and $[\bar{c}, \bar{d}]$ of $[a, b]$ and $[c, d]$ respectively, which are projective to each other in $n$ steps. If no such integer exists, then let $P([a, b],[c, d])=\infty$. We call $P([a, b],[c, d])$ the projective distance between $[a, b]$ and $[c, d]$. Thus if $P([a, b],[c, d])<\infty$, then $[a, b]$ and $[c, d]$ must be nontrivial intervals. In the four-element lattice generated by two elements, e.g. $\{a, b, a+b, a b\}$, it is easy to see that $P(a, b+$ $a],[b a, b])=1$, but $P([a, b+a],[b a, a])=\infty$.

REMARK. The notion of projective distance is the main medium used by Professor Jónsson in [8] to extend the result of Professor Grätzer in [4]. (The term "projective distance" was not used in [8] but was suggested to the author by Jónsson.) 
C. Homotheties. Given a diamond $D=[u \geqq x, y, z \geqq v]$ and an element $w$ of one of its edges, e.g. $[z, u]$, these six elements generate a finite lattice whose isomorphism type is completely determined. (For example, the sublattice generated by $D_{1}=\left[u_{1}>x_{1}, y_{1}, z_{1}>v_{1}\right]$ and $w, z_{1}<w<u_{1}$, is the lattice on the right in Fig. 5.) In any case we have two diamonds

$$
[u \geqq x+y w, y+x w, w \geqq x w+y w]
$$

and

$$
[x w+y w \geqq x w, y w, z(x w+y w) \geqq v],
$$

which we denote by $(D)_{w}$ and $\overline{(D)}_{w}$ respectively. Observing that $w$ is a term of $(D)_{w}$ even in the case it is an element of a lower edge, we have

$$
(D)_{w}=(D)_{x+y w}=\overline{(D)}_{y w} \text { and } \overline{(D)}_{w}=\overline{(D)}_{x+y w} .
$$

More generally, consider a subinterval $\left[w, w^{\prime}\right]$ of an edge of $D$, say $[z, u]$. The sublattice $\overline{\left((D)_{w}\right)_{w}}$ is defined and can be checked to be the diamond

$$
\left[x w^{\prime}+y w^{\prime} \geqq x w^{\prime}+y w, y w^{\prime}+x w, w\left(x w^{\prime}+y w^{\prime}\right) \geqq x w+y w\right] .
$$

We call it the image of $D$ under the homothety defined by $\left[w, w^{\prime}\right]$, and denoted by $(D)_{\left[w, w^{\prime}\right]}$. Clearly if $\left[w, w^{\prime}\right]$ is nontrivial, so is $(D)_{\left[w, w^{\prime}\right]}$; and if $w^{\prime}=u$ then $(D)_{\left[w . w^{\prime}\right]}$ is just $(D)_{w}$. If $z<w<w^{\prime}<u$, then the sublattice generated by $D$ and $\left\{w, w^{\prime}\right\}$ can be checked to be the lattice given in Fig. 2. However we will not need this fact, and we will therefore establish only some interesting relations between $D$ and $(D)_{\left[w, w^{\prime}\right]}$ which will be used later. For convenience, we denote $(D)_{\left[w, w^{\prime}\right]}$ by $\left[u^{\prime} \geqq x^{\prime}, y^{\prime}, x^{\prime} \geqq v^{\prime}\right]$ and

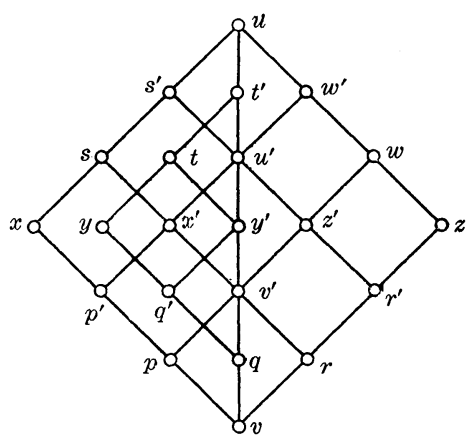

FIGURE 2 


$$
\begin{aligned}
{\left[s, s^{\prime}\right] } & =\left[x+y w, x+y w^{\prime}\right],\left[t, t^{\prime}\right]=\left[y+x w, y+x w^{\prime}\right] \\
{\left[p, p^{\prime}\right] } & =\left[x w, x w^{\prime}\right],\left[q, q^{\prime}\right]=\left[y w, y w^{\prime}\right], \\
{\left[r, r^{\prime}\right] } & =\left[z(x+y w), z\left(x+y w^{\prime}\right)\right]
\end{aligned}
$$

Then one can easily check that: The interval $\left[p, p^{\prime}\right]$ is the image of $\left[w, w^{\prime}\right]$ under the transposition of $[z, u]$ onto $[v, x]$ and

$$
\begin{gathered}
{\left[w, w^{\prime}\right] \longrightarrow\left[z^{\prime}, u^{\prime}\right] \longrightarrow\left[v^{\prime}, x^{\prime}\right] \succ\left[p, p^{\prime}\right],} \\
s^{\prime} w^{\prime}=t^{\prime} w^{\prime}=s^{\prime} t^{\prime}=u^{\prime} \\
(D)_{\left[m w^{\prime}\right]}=(D)_{\left[s, s^{\prime}\right]} .
\end{gathered}
$$

Using these preceding relations, one can extend in an obvious manner the definition of $(D)_{\left[w w^{\prime}\right]}$ to the one of $\left(M_{3^{k}}^{+}\right)_{\left[w, w^{\prime}\right]}$, where $\left[w, w^{\prime}\right]$ is a subinterval of an edge of a diamond in a string of diamonds $M_{3^{k}}^{+}$. Then $\left(M_{3^{k}}^{+}\right)_{\left[w_{\left.w^{\prime}\right]}\right.}$ is also a string of diamonds.

D. $\operatorname{Ker}\left(x_{1}, x_{2}, x_{3}\right)$. Given three elements $x_{1}, x_{2}, x_{3}$ in $L$, the sublattice generated by them is a homomorphic image of the free modular lattice of three generators $F M(3)$ (see Birkhoff [3]). It contains as a sublattice the diamond $[u \geqq x, y, z \geqq v]$ where

$$
\begin{gathered}
u=\prod_{i \neq j}\left(x_{i}+x_{j}\right) \quad v=\sum_{i \neq j} x_{i} x_{j} \\
x=\left(x_{2}+x_{3}\right)\left(x_{1}+x_{2} x_{3}\right), y=\left(x_{1}+x_{3}\right)\left(x_{2}+x_{1} x_{3}\right), \\
z=\left(x_{1}+x_{2}\right)\left(x_{3}+x_{1} x_{2}\right) .
\end{gathered}
$$

This diamond is denoted by $\operatorname{ker}\left(x_{1}, x_{2}, x_{3}\right)$. Since a homomorphic image of a transpose is also a transpose, we have the following observations:

(i) For the two element $x_{1}, x_{2}$, ker $\left(x_{1}, x_{2}, x_{3}\right)$ has the properties that its upper edge $[x, u]$ transposes up onto a subinterval $I_{1}=\left[x_{1}+\right.$ $\left.x_{3} x_{2}, x_{1}+x_{3}\left(x_{1}+x_{2}\right)\right]$ of $\left[x_{1} x_{1}+x_{2}\right]$, its lower edge $[v, x]$ (adjacent to $[x, u])$ transposes down onto a subinterval $I_{2}=\left[x_{1} x_{2}+x_{3} x_{1}, x_{1}\left(x_{2}+x_{3}\right)\right]$ of $\left[x_{1} x_{2}, x_{1}\right]$, its lower edge $[v, y]$ (opposite to $[x, u]$ ) transposes down onto a subinterval $I_{3}=\left[x_{1} x_{2}+x_{2} x_{3}, x_{2}\left(x_{3}+x_{1}\right)\right]$ of $\left[x_{1} x_{2}, x_{1}\right]$, the intervals $I_{1}$ and $I_{3}$ are the images of each other under the transposition between $\left[x_{1}, x_{1}+x_{2}\right]$ and $\left[x_{1} x_{2}, x_{2}\right]$.

(ii) If $\operatorname{ker}\left(x_{1}, x_{2}, x_{3}\right)$ is degenerate, then the sublattice generated by $x_{1}, x_{2}, x_{3}$ is distributive.

3. A diamond-normal form of projectivities. In this section we develop rather completely the notions of normality and strong normality of sequences of transposes. We first state the main theorem of the section the terminology employed here will be gradually defined.

THEOREM 3.1. Suppose $[a, b]$ and $[c, d]$ are two nontrivial intervals 
in a modular lattice $L$, and assume that

$$
P([a, b],[c, d])=n
$$

where $2<n<\infty$. Then some nontrivial subintervals $[\bar{a}, \bar{b}]$ of $[a, b]$ and $[\bar{c}, \bar{d}]$ of $[c, d]$ can be connected by a strongly normal $n+1$-termed sequence of transposes such that the associated sequence of diamonds, $D_{1}, D_{2}, D_{3}, \cdots, D_{n-1}$ has the property that for $k=1,2, \cdots, n-2$ the pairs $D_{k}, D_{k+1}$ alternately satisfy the conditions

$$
D_{k} \underset{(1)}{\longrightarrow} D_{k+1}^{*} \text { or } D_{k} \underset{(2)}{\longrightarrow} D_{k+1},
$$

and

$$
D_{k} \underset{(1)}{>} D_{k+1}^{*} \text { or } D_{k} \underset{(2)}{>} D_{k+1} \text {. }
$$

Furthermore $[\bar{a}, \bar{b}]$ and $[\bar{c}, \bar{d}]$ can be chosen so that for $k=\underline{2}, 3,4, \cdots$, $n-2, D_{k}=D_{k+1}^{*}$, whenever $D_{k}$ transposes onto $D_{k+1}^{*}$, and it can not happen that either $D_{k-1} \nearrow_{(1)} D_{k}^{*}$ and $D_{k} \searrow_{(1)} D_{k+1}^{*}$ or $D_{k-1} \searrow_{(1)} D_{k}^{*}$ and $D_{k} \nearrow_{(1)} D_{k+1}^{*}$.

REMARKs. (i) The author originally proved the theorem in a much less general form. Professor B. Jónsson suggested that he consider the theorem in this form.

(ii) Theorem 3.1 minus the last sentence is actually Theorem 1.1 in [5]. Its proof will be a combination of the proofs of Theorem 1.1 and Lemma 2.1 in [5].

3.2. Normal and strongly normal sequence of transposes.

A sequence of transposes $\left[a_{k}, b_{k}\right], 0 \leqq k \leqq n$, is said to be normal if for every $0<k<n$,

(1) either $\left[a_{k-1}, b_{k-1}\right] \succ\left[a_{k}, b_{k}\right] \succ\left[a_{k+1}, b_{k+1}\right]$ and $b_{k}=b_{k-1}+b_{k+1}$.

(2) or $\left[a_{k-1}, b_{k-1}\right] \succ\left[a_{k}, b_{k}\right] \succ\left[a_{k+1}, b_{k+1}\right]$ and $a_{k}=a_{k-1} a_{k+1}$.

If in addition, for each such $k, b_{k-1} b_{k+1} \leqq a_{k}$ in the first case and $a_{k-1}+$ $a_{k+1} \geqq b_{k}$ in the second case, then the sequence is said to be strongly normal.

In a strongly normal sequence $\left[a_{i}, b_{i}\right], 0 \leqq i \leqq n$, the sublattice generated by the six endpoints of three successive intervals, say by $a_{i}, b_{i}, i=k-1, k, k+1$, is in fact generated by three of these endpoints and is therefore finite. Moreover it is a homomorphic image of the lattice in Fig. 3 in the first case, and of its dual in the second case. Thus, it contains the diamond

$$
\left[b_{k} \geqq b_{k-1}+a_{k+1}, a_{k}, a_{k-1}+b_{k+1} \geqq a_{k-1}+a_{k+1}\right]
$$




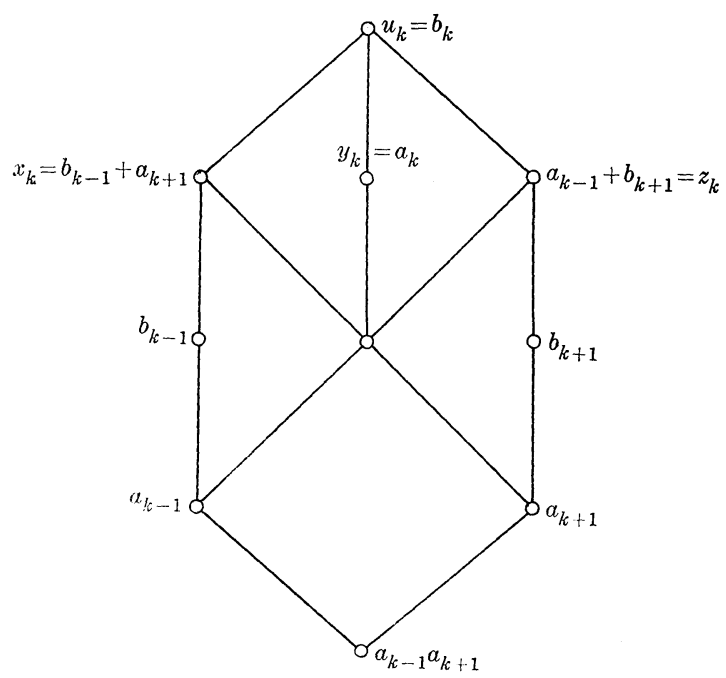

FIGURE 3

in the first case, and

$$
\left[b_{k-1} b_{k+1} \geqq a_{k-1} b_{k+1}, b_{k}, b_{k-1} a_{k+1} \geqq a_{k}\right]
$$

in the second case. We denote this diamond by

$$
D_{k}=\left[u_{k} \geqq x_{k}, y_{k}, z_{k} \geqq v_{k}\right] \text {. }
$$

Observe that in the first case

$$
\left[a_{k}, b_{k}\right]=\left[y_{k}, u_{k}\right],\left[a_{k-1}, b_{k-1}\right] \supset\left[v_{k}, x_{k}\right],\left[a_{k+1}, b_{k+1}\right]>\left[v_{k}, z_{k}\right]
$$

and in the second case

$$
\left[a_{k}, b_{k}\right]=\left[v_{k}, y_{k}\right],\left[a_{k-1}, b_{k-1}\right] \succ\left[x_{k}, u_{k}\right],\left[a_{k+1}, b_{k+1}\right]>\left[z_{k}, u_{k}\right] .
$$

The sequence $D_{1}, D_{2}, D_{3}, \cdots, D_{n-1}$ is called the associated sequence of diamonds of the given strongly normal sequence of transposes. Note that if an interval $\left[a_{k}, b_{k}\right]$ is nontrivial then all associated diamonds are nondegenerate.

In [4, Proposition 3], Grätzer showed that any two projective intervals can be connected by a normal sequence. In [8, Lemma 2] Jónsson proved a stronger version of this result, which in present terminology can be stated as follows:

Lemma 3.3. (B. Jónsson). Suppose $[a, b]$ and $[c, d]$ are nontrivial intervals in a modular lattice $L$, and assume that $P([a, b],[c, d])=n$ where $2<n<\infty$, then the following statements hold:

(i) Any normal $n+1$-termed sequence of transposes that connects $[a, b]$ and $[c, d]$ is also strongly normal. 
(ii) There are nontrivial subintervals $[\bar{a}, \bar{b}]$ of $[a, b]$ and $[\bar{c}, \bar{d}]$ of $[c, d]$ that can be connected by a strongly normal $n+1$-termed sequence of transposes.

The reader is referred to [8] for the proof.

LEMmA 3.4. If $D=[u \geqq x, y, z \geqq v]$ and $D^{\prime}=\left[u^{\prime} \geqq x^{\prime}, y^{\prime}, z^{\prime} \geqq v^{\prime}\right]$ are diamonds in $L$ with

$$
u=u^{\prime}, x \leqq x^{\prime}, y \leqq y^{\prime}, z \leqq z^{\prime},
$$

then $D^{\prime}=(D)_{x^{\prime}}=(D)_{y^{\prime}}=(D)_{z^{\prime}}$.

Proof. By symmetry we need only show that $D^{\prime}=(D)_{z^{\prime}}$. By 2.C

$$
(D)_{z^{\prime}}=\left[u \geqq x+y z^{\prime}, y+x z^{\prime}, z \geqq x z^{\prime}+y z^{\prime}\right] .
$$

We have

$$
\begin{aligned}
x z^{\prime}+y z^{\prime} & =\left(x z^{\prime}+y\right) z^{\prime}=\left(x x^{\prime} z^{\prime}+y\right) z^{\prime}=\left(x v^{\prime}+y\right) z^{\prime}=\left(x x^{\prime} y^{\prime}+y\right) z^{\prime} \\
& =\left(x y^{\prime}+y\right) z^{\prime}=(x+y) y^{\prime} z^{\prime}=u v^{\prime}=v^{\prime}, \\
x+y z^{\prime} & =x+x z^{\prime}+y z^{\prime}=x+v^{\prime}=x+x^{\prime} y^{\prime}=(x+y) x^{\prime}=u x^{\prime}=x^{\prime},
\end{aligned}
$$

and similarly $y+x z^{\prime}=y^{\prime}$. This completes the proof.

\section{COROLLARY 3.5. Suppose}

$$
\left[a_{k-1}, b_{k-1}\right]>\left[a_{k}, b_{k}\right] \supset\left[a_{k+1}, b_{k+1}\right]
$$

is a strongly normal 3-termed sequence of transposes with $D$ as its associated diamond. Suppose the points $c_{i}$ in $\left[a_{i}, b_{i}\right], i=k-1, k, k+1$, are the images of each other under the given transpositions. Then the following statements hold:

(i) The sequence

$$
\left[c_{k-1}, b_{k-1}\right]>\left[c_{k-1} c_{k+1}, b_{k}+c_{k-1} c_{k+1}\right] \supset\left[c_{k+1}, b_{k+1}\right],
$$

is a strongly normal sequence and its associated diamond is $(\bar{D})_{c_{k}}$.

(ii) If $\left[a_{k-1}^{\prime}, c_{k-1}^{\prime}\right]$ and $\left[a_{k+1}^{\prime}, c_{k+1}^{\prime}\right]$ are intervals in $L$ such that

$$
\left[a_{k-1}, c_{k-1}\right] \supset\left[a_{k-1}^{\prime}, c_{k-1}^{\prime}\right] \succ\left[a_{k}, c_{k}\right] \supset\left[a_{k+1}^{\prime}, c_{k+1}^{\prime}\right] \supset\left[a_{k+1}, c_{k+1}\right]
$$

and such that the sequence

$$
\left[a_{k-1}^{\prime}, c_{k-1}^{\prime}\right] \supset\left[a_{k}, c_{k}\right] \supset\left[a_{k+1}^{\prime}, c_{k+1}^{\prime}\right]
$$

is strongly normal, then the associated diamond of (3) is $(D)_{c_{k}}$.

Proof. It is easy to check that (2) is still strongly normal. The 
associated diamonds of (1) and (2) are

$$
\begin{aligned}
D & =\left[b_{k-1} b_{k+1} \geqq a_{k-1} b_{k+1}, b_{k}, b_{k-1} a_{k+1} \geqq a_{k}\right] \\
D^{\prime} & =\left[b_{k-1} b_{k+1} \geqq c_{k-1} b_{k+1}, b_{k}+c_{k-1} c_{k+1}, b_{k-1} c_{k+1} \geqq c_{k-1} c_{k+1}\right]
\end{aligned}
$$

and they clearly satisfy the conditions of Lemma 3.4. Therefore

$$
D^{\prime}=(D)_{b_{k-1} c_{k+1}}=\overline{(D)}_{c_{k}},
$$

by (1) in 2.C and the fact that $b_{k}\left(b_{k-1} c_{k+1}\right)=b_{k} c_{k+1}=c_{k}$.

The proof of (ii) is even more trivial.

Definition 3.6. For any diamond $D=[u \geqq x, y, z \geqq v]$ we denote the diamond $[u \geqq z, x, y \geqq v]$ by $D^{*}$.

The following lemma is crucial for the proof of Theorem 3.1.

LEMMA 3.7. Suppose $\left[a_{0}, b_{0}\right] \nearrow\left[a_{1}, b_{1}\right] \searrow\left[a_{2}, b_{2}\right] \nearrow\left[a_{3}, b_{3}\right]$ is a strongly normal sequence of transposes in $L$ with $P\left(\left[a_{0}, b_{0}\right],\left[a_{3}, b_{2}\right]\right)=3$, and consider the associated diamonds

$$
\begin{aligned}
& D_{1}=\left[u_{1}>x_{1}, y_{1}, z_{1}>v_{1}\right]\left[b_{1}>b_{0}+a_{2}, a_{1}, a_{0}+b_{2}>a_{0}+a_{2}\right] \\
& D_{2}=\left[u_{2}>x_{2} y_{2} z_{2}>v_{2}\right]\left[b_{1} b_{3}>a_{1} b_{3}, b_{2}, b_{1} a_{3}>a_{2}\right] .
\end{aligned}
$$

Then either $D_{1} \backslash D_{2}^{*}$ or else one of the following statements holds:

(i) There exists $c_{0}$ with $a_{0} \leqq c_{0}<b_{0}$ such that if $c_{i}, 1 \leqq i \leqq 3$, is its image in $\left[a_{i}, b_{i}\right]$ under the given transpositions then

$$
\left[c_{0}, b_{0}\right] \succ\left[c_{1}, d_{1}\right] \longrightarrow\left[c_{1} c_{3}, c_{1} c_{3}+b_{2}\right] \succ\left[c_{3}, b_{3}\right]
$$

is a strongly normal sequence of transposes, its associated diamonds

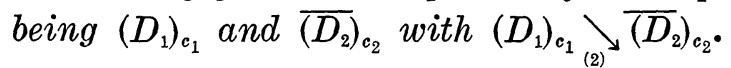

(ii) There exists $c_{0}$ with $a<c_{0} \leqq b$ such that if $c_{i}, 1 \leqq i \leqq 3$, is its image in $\left[a_{i}, b_{i}\right]$ under the given transpositions then

$$
\left.\left[a_{0}, c_{0}\right] \succ\left[c_{0}+c_{2}\right],\left(c_{0}+c_{2}\right) a_{1}\right] \longrightarrow\left[a_{2}, c_{2}\right] \succ\left[a_{3}, c_{3}\right]
$$

is a strongly normal sequence of transposes, its associated diamonds being $\overline{\left(D_{1}\right)_{c_{1}}}$ and $\left(D_{2}\right)_{c_{2}}$ with $\overline{(D)_{c_{1}}} \searrow_{(2)}\left(D_{2}\right)_{c_{2}}$.

Proof. Observing that

$$
v_{1}+y_{2}=z_{1}, u_{2} y_{1}=x_{2} \text { and } z_{1} \leqq v_{1}+u_{2} \leqq u_{1}, v_{2} \leqq u_{2} v_{1} \leqq x_{2},
$$

we consider three cases.

Case 1. $v_{1}+u_{2}=u_{1}$ and $v_{1} u_{2}=v_{2}$. Thus we have

$$
\left[v_{2}, u_{2}\right] \longrightarrow\left[v_{1}, u_{1}\right] \text {. }
$$


Since $v_{1}+y_{2}=z_{1}$ and $u_{2} y_{1}=x_{2}$, the transposition (2) maps $u_{2}, x_{2}, y_{2}, v_{2}$ into $u_{1}, y_{1}, z_{1}, v_{1}$ respectively, and hence we have the diamond [ $u_{1}>$ $\left.z_{2}+v_{1}, y_{1}, z_{1}>v_{1}\right]$ with

$$
\left[u_{1}>z_{2}+v_{1}, y_{1}, z_{1}>v_{1}\right]_{(1)}\left[u_{2}>z_{2}, x_{2}, y_{2}>v_{2}\right] \text {. }
$$

If $z_{2}+v_{1}=x_{1}$, then $D_{1}$ transposes down onto $D_{2}^{*}$, i.e., $D_{1} \backslash D_{2}^{*}$. Suppose on the contrary that $z_{2}+v_{1} \neq x_{1}$. We claim that this leads to a contradiction to the hypothesis $P\left(\left[a_{0}, b_{0}\right],\left[a_{3}, b_{3}\right]\right)=3$. Our reasoning is motivated by Fig. 4. Let $x_{1}^{\prime}=z_{2}+v_{1}$ First we observe that $x_{1}^{\prime}$ and $x_{1}$, having the same relative complement $y_{1}$ in $\left[v_{1}, u_{1}\right]$, are therefore incomparable to each other. It follows that $\left[x_{1} x_{1}^{\prime}, x_{1}\right]$ and $\left[x_{1}^{\prime}, x_{1}+x_{1}^{\prime}\right]$ are nontrivial subintervals of $\left[v_{1}, x_{1}\right]$ and $\left[x_{1}^{\prime}, u_{1}\right]$ respectively, and

$$
\left[x_{1} x_{1}^{\prime}, x_{1}\right] \succ\left[x_{1}^{\prime}, x_{1}+x_{1}^{\prime}\right] \text {. }
$$

Since $u_{1} b_{3}=u_{2}$ and $\left[x_{1}^{\prime}, u_{1}\right] \searrow\left[z_{2}, u_{2}\right] \nearrow\left[a_{3}, b_{3}\right]$, the dual of the Transposition Lemma applies, and we have

$$
\left[x_{1}^{\prime}, u_{1}\right] \succ\left[x_{1}^{\prime}+a_{3}, x_{1}^{\prime}+b_{3}\right] \longrightarrow\left[a_{3}, b_{3}\right] \text {. }
$$

It follows from (4) and (5) that the nontrivial subinterval $\left[b_{0} x_{1}^{\prime}, b_{0}\right]$ of $\left[a_{0}, b_{0}\right]$ transposes up onto a subinterval of $\left[x_{1}^{\prime}+a_{3}, x_{1}^{\prime}+b_{3}\right]$, which

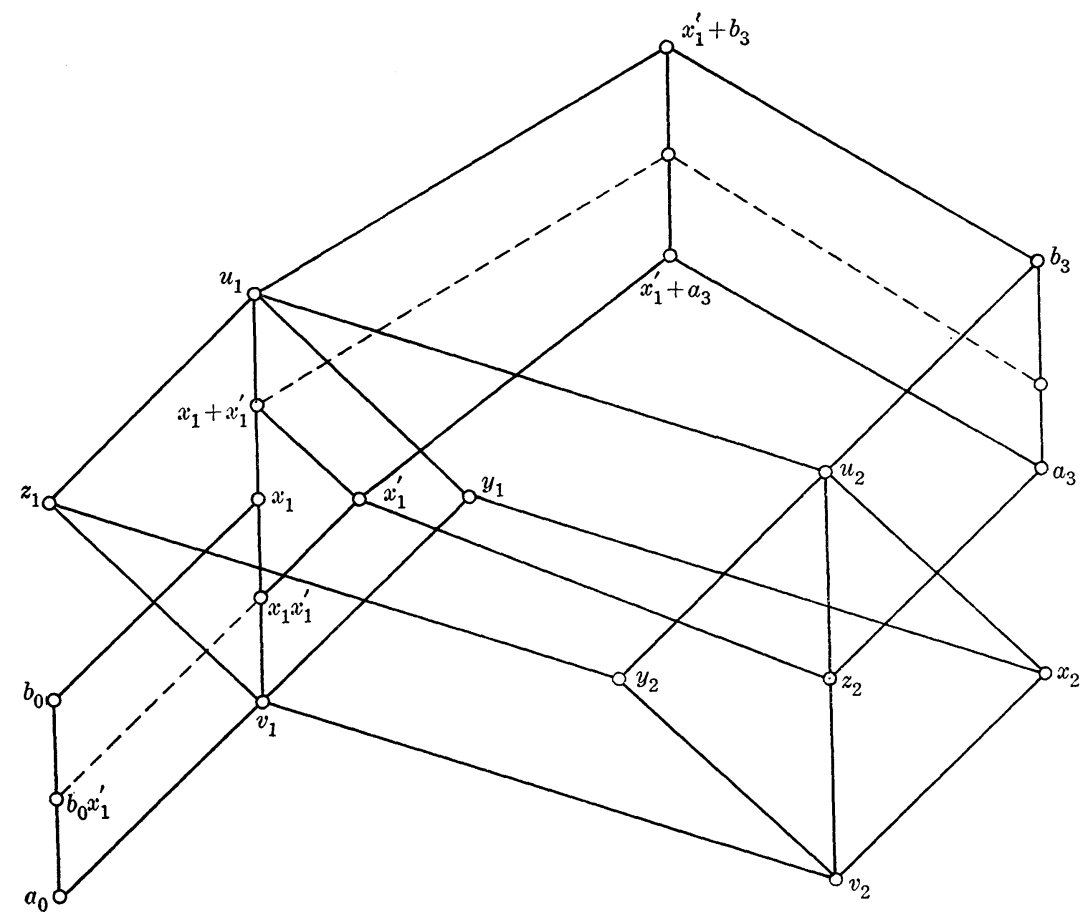

FIGURE 4 
transposes down onto a subinterval of $\left[a_{3}, b_{3}\right]$. Thus $P\left(\left[a_{0}, b_{0}\right],\left[a_{3}, b_{3}\right]\right) \leqq$ 2 , and we have a contradiction. Therefore $D_{1} \backslash D_{2}^{*}$ in this case.

Case 2. $v_{1}+u_{2}<u_{1}$. Our reasoning is motivated by Fig. 5. Let $w=v_{1}+u_{2}$ and $c_{1}=y_{1}+x_{1} w$, and for $i=0,2,3$, let $c_{i}$ be the member of $\left[a_{i}, b_{i}\right]$ corresponding to $c_{1}$ under the given transpositions. The sequence of transposes

$$
\left.\left.\left[c_{0}, b_{0}\right] \supset\left[c_{1}, b_{1}\right] \succ\right] c_{1} c_{3}, b_{2}+c_{1} c_{3}\right] \supset\left[c_{3}, b_{3}\right]
$$

is clearly normal, and since $\left[c_{1}, b_{1}\right]$ is nontrivial

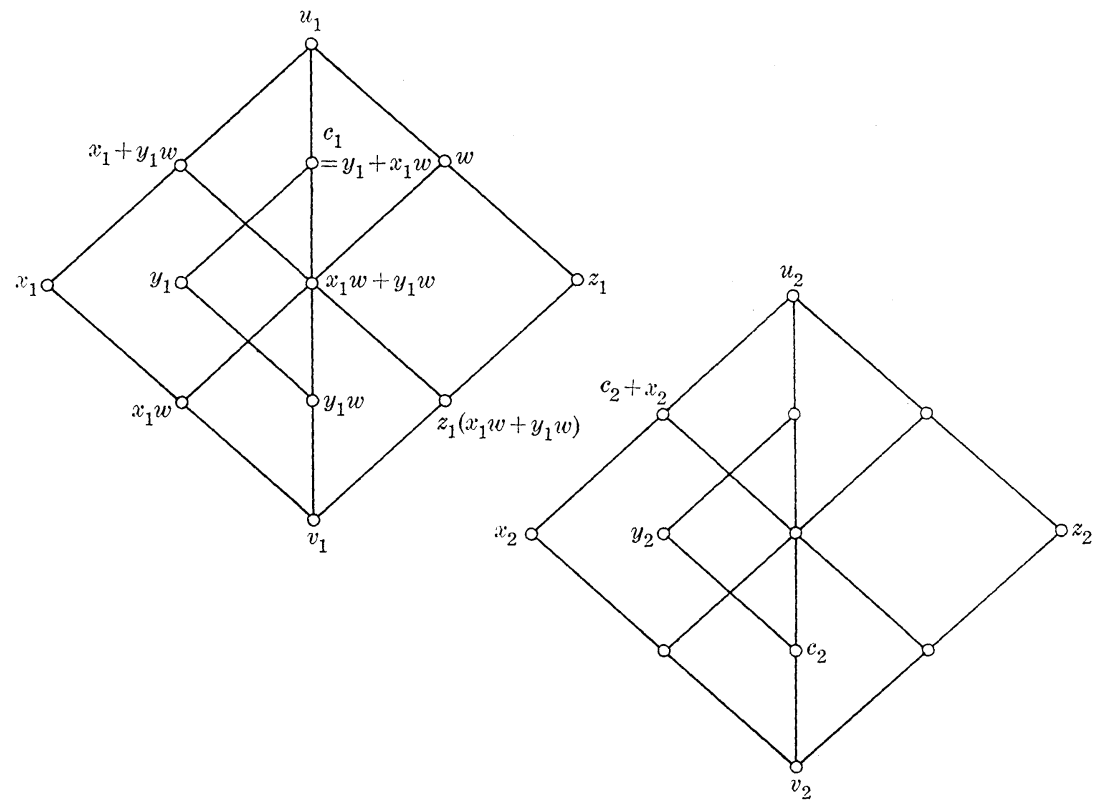

FIGURE 5

$$
3 \geqq P\left(\left[c_{0}, b_{0}\right],\left[c_{3}, b_{3}\right]\right) \geqq P\left(\left[a_{0}, b_{0}\right],\left[a_{3}, b_{3}\right]\right)=3 .
$$

It follows from Lemma 3.3 that (6) is strongly normal. By Corollary 3.5 and its dual, the two associated diamonds are

$$
\begin{aligned}
& \left(D_{1}\right)_{c_{1}}=\left(D_{1}\right)_{w}=\left[u_{1}>x_{1}+y_{1} w, c_{1}, w>x_{1} w+y_{1} w\right] \\
& \left(D_{2}\right)_{c_{2}}=\left[u_{2}>c_{2}+x_{2}, b_{2}+c_{1} c_{3}, c_{2}+z_{2}>c_{1} c_{3}\right] .
\end{aligned}
$$

Following the ideas of Jónsson in [8, Lemma 3], we infer that

$$
\left[x_{1} w+y_{1} w, w\right]>\left[c_{2}+x_{2}, u_{2}\right] .
$$

In fact, from the observation that $u_{2} \leqq w \leqq\left(x_{1}+u_{2}\right)\left(y_{1}+u_{2}\right)$ and $x_{2} \leqq$ $y_{1}$, we have 


$$
\begin{aligned}
\left(x_{1} w+y_{1} w\right)+u_{2} & =\left(x_{1}+u_{2}\right) w+\left(y_{1}+u_{2}\right) w=w, \\
\left(x_{1} w+y_{1} w\right) u_{2} & =u_{2} w\left(x_{1} w+y_{1}\right)=u_{2}\left(x_{1} w+y_{1}\right) \\
& =\left(x_{2}+y_{2}\right)\left(x_{1} w+y_{1}\right) \\
& =x_{2}+y_{2}\left(x_{1} w+y_{1}\right)=x_{2}+b_{2} c_{1}=x_{2}+c_{2} .
\end{aligned}
$$

Thus (i) holds in this case.

Case 3. $v_{2}<v_{1} u_{2}$. Applying the dual of Case 1 with a reversal in the order of the intervals $\left[\alpha_{i}, b_{i}\right]$, we see that (ii) holds in this case.

The three preceding cases cover all possibilities. The proof is therefore complete.

Lemma 3.8. Suppose $D_{i}=\left[u_{i} \geqq x_{i}, y_{i}, z_{i} \geqq v_{i}\right], i=1,2$ are two diamonds in $L$, and assume that one of the following conditions holds

$$
D_{1} \underset{(2)}{D_{11}} D_{2}^{*} \text {. }
$$

Let $c_{1}$ be a member of $\left[v_{1}, y_{1}\right]$ and $c_{2}$ its image in $\left[y_{2}, u_{2}\right]$ under the transposition of $\left[v_{1}, y_{1}\right]$ onto $\left[y_{2}, u_{2}\right]$. Then we have
(i) ${\overline{\left(D_{1}\right)}}_{c_{1}} \nearrow\left(D_{2}^{*}\right)_{c_{2}}$ if (1) holds
(ii) $\overline{\left(D_{1}\right)_{c_{1}}} \nearrow_{(2)}\left(D_{2}\right)_{c_{2}}$ if (2) holds.

Proof. Assume that (2) holds. It is clear that the first lower edge of $\left(D_{2}\right)_{c_{2}}$ transposes down onto $\left[x_{2} c_{2}, x_{2}\right]$, hence onto $\left[u_{1} c_{2}, u_{1}\right]$. Therefore

$$
\left(D_{1}\right)_{u_{1} c_{2}} \underset{(2)}{\longrightarrow}\left(D_{2}\right)_{c_{2}} \text {. }
$$

Since $c_{1}=c_{2} y_{1}=c_{2} u_{1} y_{1}$, we infer from the formula (1) in 2.C that $\overline{\left(D_{1}\right)_{c_{1}}}=\left(D_{1}\right)_{u_{1} c_{2}}$ and therefore $\overline{\left(\overline{D_{1}}\right)_{c_{1}}} \nearrow_{(2)}\left(D_{2}\right)_{c_{2}}$.

Suppose (1) holds. We similarly have $\overline{\left(\overline{D_{1}}\right)_{c_{1}}}=\left(D_{1}\right)_{u_{1} c_{2}}$. Furthermore the transposition of $\left[v_{1}, u_{1}\right]$ onto $\left[v_{2}, u_{2}\right]$ maps $u_{1} c_{2}$ into $c_{2}$, hence $\left(D_{1}\right)_{u_{1} c_{2}}$ onto $\left(D_{2}^{*}\right)_{c_{2}}$. Thus

$$
\left(\overline{\left.D_{1}\right)_{c_{1}}}=\left(D_{1}\right)_{u_{1} c_{2}} \underset{(1)}{\longrightarrow}\left(D_{2}^{*}\right)_{c_{2}}\right.
$$

and therefore (i) holds.

REMARK. In Lemma 3.8 if we replace the hypothesis $D_{1} \nearrow D_{2}^{*}$ by $D_{1}=D_{2}^{*}$, then clearly we have the conclusion that $\overline{\left(D_{1}\right)_{c_{1}}}=\left(D_{2}^{*}\right)_{c_{2}}$. 
3.9. Proof of Theorem 3.1. We prove the theorem by induction on $n$. For $n=3$, the theorem holds by Lemma 3.7 and its dual. So assume that $3<n=P([a, b],[c, d])<\infty$, and the theorem holds with $n$ replaced by $n-1$. Then there are nontrivial subintervals [ $a^{*}$, $\left.b^{*}\right]$ of $[a, b]$ and $\left[c^{*}, d^{*}\right]$ of $[c, d]$ that are projective to each other in $n$ steps. By duality we may assume that $\left[a^{*}, b^{*}\right]$ transposes up onto an interval $\left[a^{\prime}, b^{\prime}\right]$ that can be projective to $\left[c^{*}, d^{*}\right]$ in $n-1$ steps. By the inductive hypothesis, we may assume furthermore that $\left[a^{\prime}, b^{\prime}\right]$ and $\left[c^{*}, d^{*}\right]$ are connected by a strongly normal sequence of transposes

$$
\left[a^{\prime}, b^{\prime}\right] \succ\left[a_{2}, b_{2}\right] \supset\left[a_{3}, b_{3}\right] \cdots\left[a_{n}, b_{n}\right]=\left[c^{*}, d^{*}\right]
$$

such that the associated sequence of diamonds, denoted by $D_{i}=\left[u_{i}\right\rangle$ $\left.x_{i}, y_{i}, z_{i}>v_{i}\right], 2 \leqq i \leqq n-1$, satisfies the conclusion of the theorem. Let $\left[a_{0}, b_{0}\right]=\left[a^{*}, b^{*}\right]$ and $\left[a_{1}, b_{1}\right]=\left[a^{\prime}\left(b^{*}+b_{2}\right), b^{*}+b_{2}\right]$ We easily see that the sequence

$$
\left[a^{*}, b^{*}\right]=\left[a_{0}, b_{0}\right] \longrightarrow\left[a_{1}, b_{2}\right] \succ\left[a_{2}, b_{2}\right] \succ \cdots\left[a_{n}, b_{n}\right]=\left[c^{*}, d^{*}\right]
$$

is normal, and therefore strongly normal by Lemma 3.3. If $D_{1}$ is the associated diamond of $\left[a_{0}, b_{0}\right] \nearrow\left[a_{1}, b_{1}\right] \searrow\left[{ }_{2} a, b_{2}\right]$, then the diamonds associated with (2) are $D_{1}, D_{2}, D_{3}, \cdots, D_{n-1}$. As regards the second diamond $D_{2}$, this follows from Corollary 3.5 (ii), and for the other it is obvious.

We now apply Lemma 3.7 to the first four intervals in (2).

If $D_{1} \backslash D_{2}^{*}$ we claim that $D_{2} \nearrow D_{3}$, and the conclusions of the theorem therefore hold in this case. Otherwise $D_{2} \nearrow D_{3}^{*}$ and hence

$$
\left[z_{1}, u_{1}\right] \succ\left[y_{2}, u_{2}\right] \underset{(1)}{\longrightarrow}\left[x_{3}, u_{3}\right] \cdot
$$

Then following the idea of Jónsson in [8, Lemma 4] we infer that

$$
\begin{aligned}
& {\left[a_{0}, b_{0}\right] \succ\left[v_{1}, x_{1}\right] \succ\left[z_{1}, u_{1}\right] \succ\left[z_{1}+x_{3}, u_{1}+x_{3}\right]>} \\
& {\left[x_{3}, u_{3}\right] \succ\left[v_{3}, z_{3}\right] \succ\left[a_{4}, b_{4}\right] .}
\end{aligned}
$$

In fact, the first two and the last two transpositions are clearly true. . Since $u_{1} u_{3}=u_{2}$, we can apply the dual of the Transposition Lemma to the sequence (3) to infer that the third and the fourth transpositions hold. Thus (4) holds and consequently $\left[a_{0}, b_{0}\right]$ and $\left[a_{4}, b_{4}\right]$ can be connected in two steps which clearly contradicts the hypothesis that $P([a, b]$, $[c, d])=n$.

So we can assume that either (i) or (ii) of Lemma 3.7 applies, and we can choose $c_{0} \in\left[a_{0}, b_{0}\right]$ accordingly. Let $c_{1}, c_{2}, \cdots, c_{n}$ be the image of $c_{0}$ under the given transpositions. Assume first that 3.7 (i) applies. From the sequence 
(5)

$$
\left[c_{0}, b_{0}\right] \supset\left[c_{1}, b_{1}\right]>\left[c_{2}, b_{2}\right] \succ \cdots\left[a_{n}, b_{n}\right] .
$$

We obtain a normal sequence of transposes

$$
\left.\left[c_{0}^{\prime}, b_{0}^{\prime}\right] \succ\left[c_{1}^{\prime}, b_{1}^{\prime}\right] \succ_{2}^{\prime}, b_{2}^{\prime}\right] \succ \cdots\left[a_{n}^{\prime}, b_{n}^{\prime}\right]
$$

by letting $\left[c_{k}^{\prime}, b_{k}^{\prime}\right]=\left[c_{k-1} c_{k+1}, b_{k}+c_{k-1} c_{k-1}\right]$ for $k$ even with $0<k<n$, and $\left[c_{k}^{\prime}, b_{k}^{\prime}\right]=\left[c_{k}, b_{k}\right]$ in other cases. The sequence (6) is strongly normal by Lemma 3.3. Let $D_{k}^{\prime}=\left[u_{k}^{\prime}>x_{k}^{\prime}, y_{k}^{\prime}, z_{k}^{\prime}>v_{k}^{\prime}\right], k=1,2,3, \cdots$, $n-1$ be the sequence of diamonds associated with (6). By Corollary 3.5 and its dual,

$$
D_{k}^{\prime}=\left(D_{k}\right)_{c_{k}}
$$

if $k$ is odd, and

$$
D_{k}^{\prime}=\left(\overline{D_{k}}\right)_{c_{k}}
$$

in other cases.

Thus, the associated sequence of diamonds of (6), $D_{1}^{\prime}, D_{2}^{\prime}, \cdots, D_{n-1}^{\prime}$, is the following sequence

$$
\left(D_{1}\right)_{c_{1}},{\overline{\left(D_{2}\right)}}_{c_{2}}, \cdots,\left(D_{2 k-1}\right)_{c_{2 k-1}},{\overline{\left(D_{2 k}\right)_{c_{2 k}}}}, \cdots
$$

Since we assume that 3.7(i) holds, we have furthermore

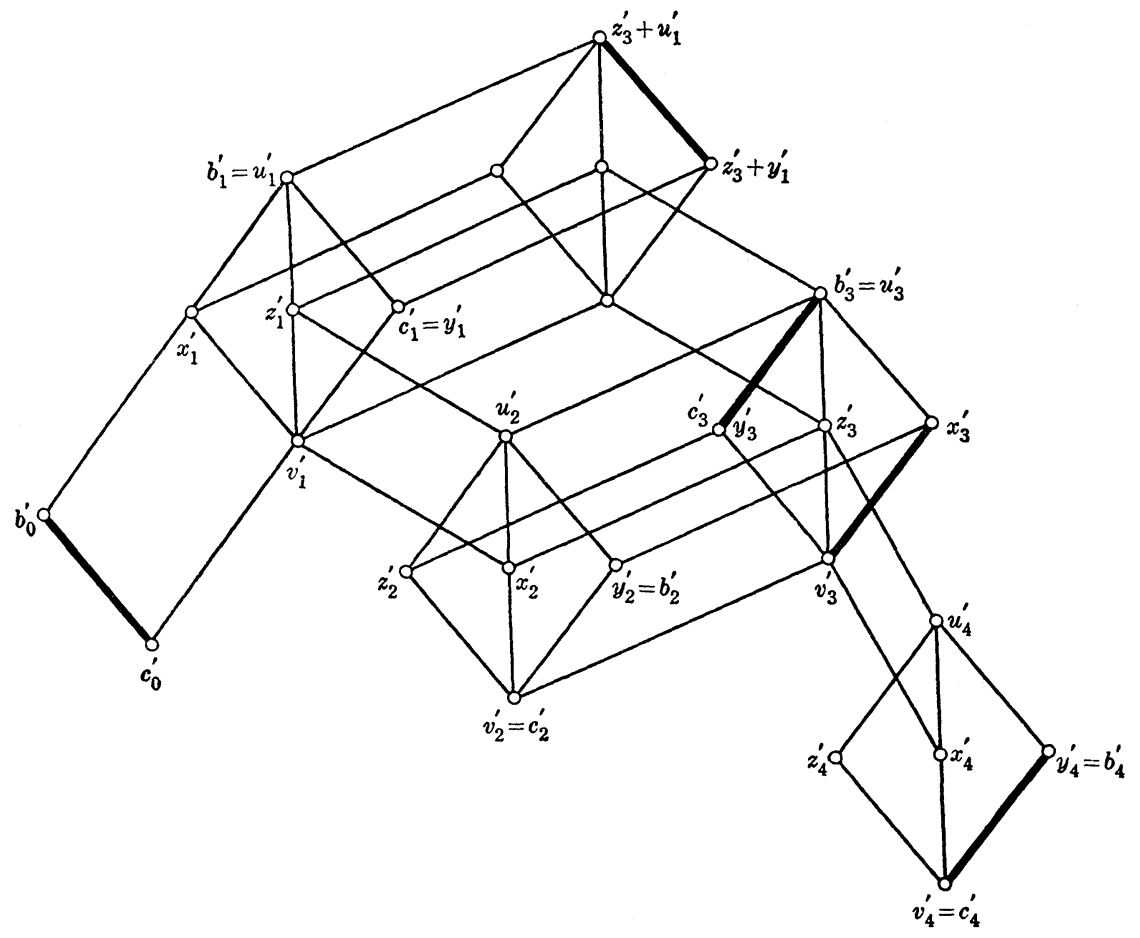

FigURE 6 


$$
\left(D_{1}\right)_{c_{1}} \underset{(2)}{\longrightarrow} \overline{\left(D_{2}\right)_{c_{2}}} \text {, i.e., } D_{1}^{\prime} \varlimsup_{(2)} D_{2}^{\prime} \text {. }
$$

Therefore if $D_{2} / D_{3}$, then in view of (8), Lemma 3.8 and its dual, we conclude that the sequence (7) associated with (6) satisfies the conclusions of the theorem. It remains only to consider the case $D_{2} \nearrow_{(1)} D_{3}^{*}$. Again by Lemma 3.8 we have $D_{2}^{\prime} \nearrow_{(1)} D_{3}^{\prime *}$. Our reasoning is motivated by Fig. 6 . Since $u_{1}^{\prime} u_{3}^{\prime}=u_{2}^{\prime}$ (from the definition of associated diamonds), we apply the dual of the Transposition Lemma to the diamond $D_{1}^{\prime}$ and the sequence $\left[v_{1}^{\prime}, z_{1}^{\prime}\right] \searrow\left[x_{2}^{\prime}, u_{2}^{\prime}\right] \nearrow\left[z_{3}^{\prime}, u_{3}^{\prime}\right]$ and we have a diamond $z_{3}^{\prime}+D_{1}^{\prime}$ with the lower edge $\left[z_{3}^{\prime}+v_{1}^{\prime}, z_{3}^{\prime}+z_{1}^{\prime}\right]$ transposing down onto $\left[z_{3}^{\prime}, u_{3}^{\prime}\right]$. Using the strong normality of (6), one can check that the sequence

$$
\left.\left[c_{0}^{\prime}, b_{0}^{\prime}\right] \succ\left[z_{3}^{\prime}+y_{1}^{\prime}, z_{3}^{\prime}+u_{1}^{\prime}\right] \supset\left[v_{3}^{\prime}, x_{3}^{\prime}\right] \supset\left[c_{3}^{\prime}, b_{3}^{\prime}\right] \succ_{4}^{\prime}, b_{4}^{\prime}\right]
$$

is strongly normal. The associated diamonds of (9) are $z_{3}^{\prime}+D_{1}^{\prime},\left(D_{3}^{\prime}\right)^{*}$, $D_{3}^{\prime}$. Observe that $D_{3} \searrow D_{4}$, since $D_{2} \nearrow_{(1)} D_{3}^{*}$. Hence $D_{3}^{\prime} \searrow_{(2)} D_{4}^{\prime}$. Thus if we replace the second and the third terms in (6) by the corresponding terms in (9) we obtain a strongly normal sequence that connects the nontrivial subintervals $\left[c_{0}^{\prime}, b_{0}^{\prime}\right]$ and $\left[c_{n}^{\prime}, b_{n}^{\prime}\right]$ with the associated sequence of diamonds

$$
z_{3}^{\prime}+D_{1}^{\prime},\left(D_{3}^{\prime}\right)^{*}, D_{3}^{\prime}, D_{4}^{\prime}, \cdots, D_{n-1}^{\prime},
$$

which clearly satisfies the conclusion of the theorem.

The proof for the case when 3.7(ii) applies can be done in a similar way. This completes the proof of Theorem 3.1.

4. Proof of the Main Lemma. The proof of the Main Lemma is based upon a series of lemmas.

LEMMA 4.1. Let a strongly normal four-termed sequence of transposes

$$
\left[a_{0}, b_{0}\right] \supset\left[a_{1}, b_{1}\right] \longrightarrow\left[a_{2}, b_{2}\right] \succ\left[a_{3}, b_{3}\right]
$$

be given in $L$, with $\left[a_{0}, b_{0}\right]$ nontrivial and $b_{0} \leqq a_{3}$. If $D_{1}$ and $D_{2}$ are the associated diamonds, then it cannot happen that $D_{1} \underset{(2)}{\searrow} D_{2}$, and the condition $D_{1} \searrow_{(1)} D_{2}^{*}$ implies that $D_{1}=D_{2}^{*}$.

Proof. The associated diamonds $D_{1}, D_{2}$ are

$$
\begin{aligned}
& D_{1}=\left[u_{1}>x_{1}, y_{1}, z_{1}>v_{1}\right]=\left[b_{1}>b_{0}+a_{2}, a_{1}, a_{0}+b_{2}>a_{0}+a_{2}\right], \\
& D_{2}=\left[u_{2}>x_{2}, y_{2}, z_{2}>v_{2}\right]=\left[b_{1} b_{3}>a_{1} b_{3}, b_{2}, b_{1} a_{3}>a_{2}\right] .
\end{aligned}
$$


Suppose first $D_{1} \backslash D_{2}^{*}$. In this case, using the hypothesis that $b_{0} \leqq a_{3}$ we have

$$
u_{1}=x_{1}+z_{1}=\left(b_{0}+a_{2}\right)+\left(a_{0}+b_{2}\right) \leqq b_{3},
$$

therefore $u_{1}=u_{1} b_{3}=u_{2}$, and this readily implies that $D_{1}=D_{2}^{*}$, since $D_{1} \searrow D_{2}^{*}$.

Next assume $D_{1} \backslash D_{2}$. We claim that this leads to a contradiction and hence completes the proof. In this case, also using the hypothesis that $b_{0} \leqq a_{3}$ we have

$$
\begin{aligned}
u_{2} \leqq u_{1} & =b_{0}+z_{1}=b_{0}+v_{1}+z_{2} \\
& =b_{0}+\left(a_{0}+a_{2}\right)+z_{2}=b_{0}+z_{2} \leqq a_{3},
\end{aligned}
$$

therefore $u_{2}=u_{2} a_{3}=z_{2}$ which clearly contradicts the hypothesis that $\left[a_{0}, b_{0}\right]$ is nontrivial. The lemma is proved.

Lemma 4.2. Suppose

$$
A_{1}, A_{2}, A_{3} \notin \boldsymbol{H S}(L)
$$

and suppose $[a, b]$ and $[c, d]$ are nontrivial intervals in $L$ such that $P([a, b],[c, d])=n<\infty$. Then $n \leqq 5$. Furthermore if $b \leqq c$, then there exist nontrivial subintervals $[\bar{a}, \bar{b}]$ and $[\bar{c}, \bar{d}]$ of $[a, b]$ and $[c, d]$ respectively, such that one of the four following conditions holds:

(i) $n=3$, and there is a diamond $[u>x, y, z>v]$ with

$$
[\bar{a}, \bar{b}] \supset[v, x] \text { and }[x, u] \longrightarrow[\bar{c}, \bar{d}] \text {. }
$$

(ii) $n=4$, and there exist two diamonds $D_{i}^{\prime}=\left[u_{i}^{\prime}>x_{i}^{\prime}, y_{i}^{\prime}, z_{i}^{\prime}>v_{i}\right]$, $i=1,2$, such that $D_{1}^{\prime} \nearrow_{(2)} D_{2}^{\prime}$ and

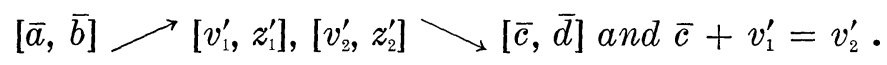

(iii) $n=4$, and there exist two diamonds $D_{i}^{\prime}=\left[u_{i}^{\prime}>x_{i}^{\prime}, y_{i}^{\prime}, z_{i}^{\prime}>v_{i}^{\prime}\right]$, $i=1,2$, such that $D_{1}^{\prime} \nearrow_{(2)} D_{2}^{\prime}$ and

$$
[\bar{a}, \bar{b}] \succ\left[x_{1}^{\prime}, u_{1}^{\prime}\right],\left[x_{2}^{\prime}, u_{2}^{\prime}\right] \supset[\bar{c}, \bar{d}] \text { and } \bar{b} u_{2}^{\prime}=u_{1}^{\prime} \text {. }
$$

(iv) $n=5$, and there exist two diamonds $D_{i}^{\prime}=\left[u_{i}^{\prime}>x_{i}^{\prime}, y_{i}^{\prime}, z_{i}^{\prime}>v_{i}^{\prime}\right]$, $i=1,2$, such that $D_{2}^{\prime} \underset{(2)}{\searrow} D_{1}^{\prime}$ and

$$
[\bar{a}, \bar{b}] \supset\left[v_{1}^{\prime}, x_{1}^{\prime}\right],\left[z_{2}^{\prime}, u_{2}^{\prime}\right] \supset[\bar{c}, \bar{d}] \text {. }
$$

Proof. We may assume that $n>2$, and by Theorem we may therefore assume that some nontrivial subintervals $[\bar{a}, \bar{b}]$ of $[a, b]$ and $[\bar{c}, \bar{d}]$ of $[c, d]$ can be connected by a strongly normal $n+1$-termed 

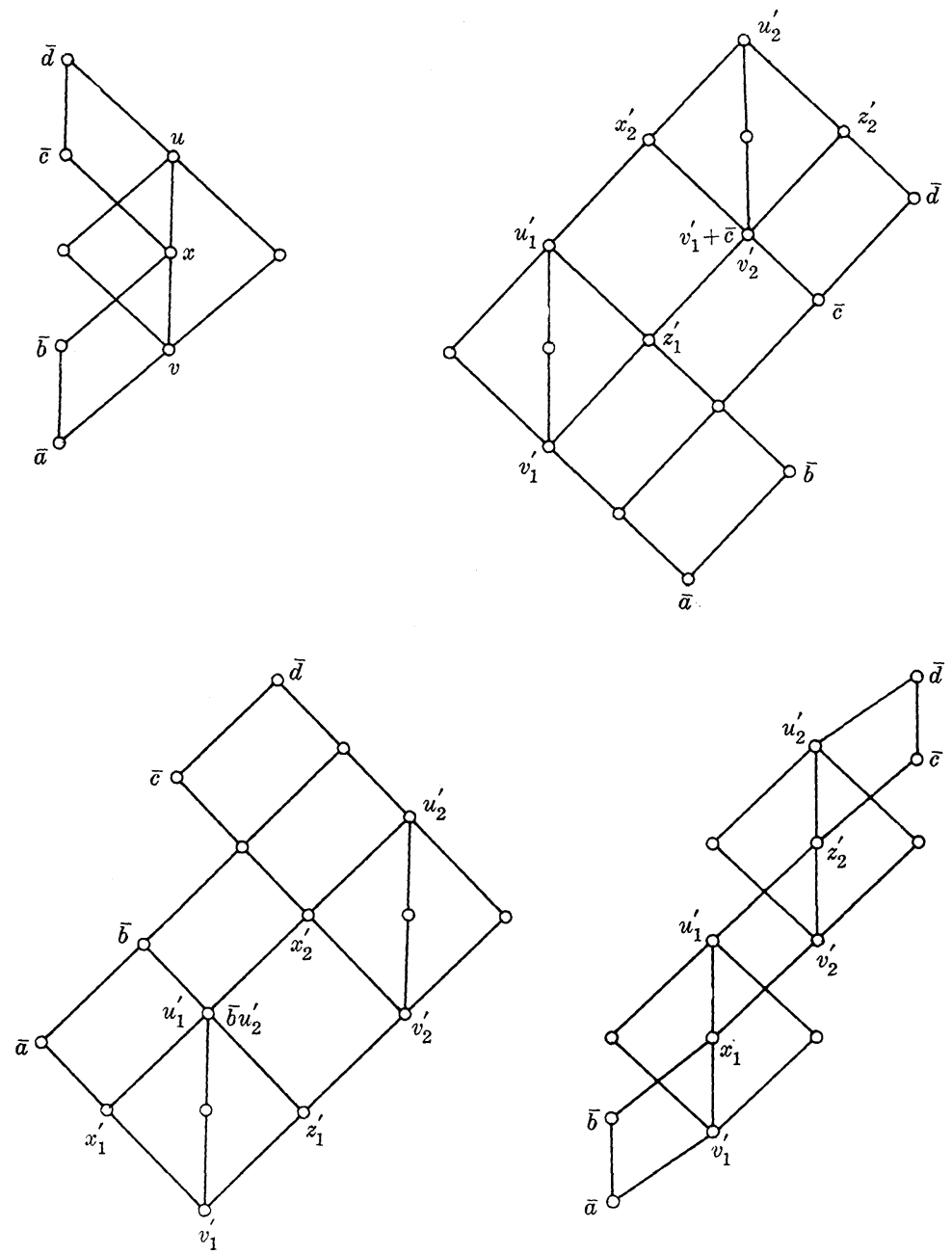

Figure 7

sequence of transposes such that the associated sequence of diamonds satisfies the conclusion of that theorem. We prove the first part of the theorem by showing that the assumption $n=6$ leads to a contradiction. By duality we may assume that

$$
\begin{aligned}
& {[\bar{a}, \bar{b}]=\left[a_{0}, b_{0}\right] \supset\left[a_{1}, b_{1}\right] \succ\left[a_{2}, b_{2}\right] \supset\left[a_{3}, b_{3}\right]>\left[a_{4}, b_{4}\right]} \\
& \longrightarrow\left[a_{5}, b_{5}\right] \succ\left[a_{6}, b_{6}\right]=[\bar{c}, \bar{d}]
\end{aligned}
$$

is the given sequence of transposes. The diamonds associated with this sequence are

$$
\begin{aligned}
& D_{1}=\left[u_{1}>x_{1}, y_{1}, z_{1}>v_{1}\right]=\left[b_{1}>b_{0}+a_{2}, a_{1}, a_{0}+b_{2}>a_{0}+a_{2}\right] \\
& D_{2}=\left[u_{2}>x_{2}, y_{2}, z_{2}>v_{2}\right]=\left[b_{1} b_{3}>a_{1} b_{3}, b_{2}, b_{1} a_{3}>a_{2}\right] \\
& D_{3}=\left[u_{3}>x_{3}, y_{3}, z_{3}>v_{3}\right]=\left[b_{3}>b_{2}+a_{4}, a_{3}, a_{2}+b_{4}>a_{2}+a_{4}\right]
\end{aligned}
$$




$$
\begin{aligned}
& D_{4}=\left[u_{4}>x_{4}, y_{4}, z_{4}>v_{4}\right]=\left[b_{3} b_{5}>a_{3} b_{5}, b_{4}, b_{3} a_{5}>a_{4}\right] \\
& D_{6}=\left[u_{5}>x_{5}, y_{5}, z_{5}>v_{5}\right]=\left[b_{5}>b_{4}+a_{6}, a_{5}, a_{4}+b_{6}>a_{4}+a_{6}\right] .
\end{aligned}
$$

Consider any three successive diamonds, say $D_{1}, D_{2}$, and $D_{3}$. If $D_{1} \searrow_{(2)} D_{2}$ and $D_{2} \nearrow_{(2)} D_{3}$, then we have a contradiction. In fact, since $u_{1} u_{3}=u_{2}$ therefore in this case the set

$$
B=D_{1} \cup D_{2} \cup D_{3} \cup\left\{v_{1}+v_{3}, v_{1}+u_{3}, v_{3}+u_{1}, u_{1}+u_{3}\right\}
$$

is easily seen to be a sublattice of $L$ with $A_{2}$ as a homomorphic image, in contradiction to the hypothesis that $A_{2} \notin \boldsymbol{H S}(L)$. Therefore one of the two following conditions must hold

$$
\begin{aligned}
& D_{1} \underset{(1)}{\longrightarrow} D_{2}^{*}, D_{2} \underset{(2)}{\longrightarrow} D_{3}, D_{3}=D_{4}^{*} \text { and } D_{4} \underset{\text { (2) }}{\longrightarrow} D_{5} \\
& D_{1} \underset{\text { (2) }}{\longrightarrow} D_{2}, D_{2}=D_{3}^{*}, D_{3} \underset{\text { (2) }}{\longrightarrow} D_{4} \text { and } D_{4}=D_{5}^{*} .
\end{aligned}
$$

If (1) holds, then $D_{2} \cup D_{3} \cup D_{5}$ is a sublattice of $L$ which has $A_{1}$ as a homomorphic image, contradicting the hypothesis of the theorem. If (2) holds, we arrive at a contradiction in a similar manner by considering the sublattice $D_{1} \cup D_{2} \cup D_{4}$. Thus the assumption $n \geqq 6$ always leads to a contradiction.

Now assume that $b \leqq c$. Then $\bar{b} \leqq \bar{c}$. Therefore the case $n \leqq 2$ is clearly excluded, and we must have $n=3,4$, or 5 .

Suppose $n=3$. Then $[\bar{a}, \bar{b}]$ and $[\bar{c}, \bar{d}]$ are connected by a strongly normal four-termed sequence of transposes,

$$
[\bar{a}, \bar{b}]=\left[\bar{a}_{0}, \bar{b}_{0}\right] \succ\left[\bar{a}_{1}, \bar{b}_{1}\right] \longrightarrow\left[\bar{a}_{2}, \bar{b}_{2}\right] \succ\left[a_{3}, b_{3}\right]=[\bar{c}, \bar{d}],
$$

with the associated diamonds $D_{1}, D_{2}$ satisfying either $D_{1} \searrow D_{2}^{*}$ or $D_{1} \backslash D_{2}$. (The dual form of (3) is excluded by the condition $\bar{b} \leqq \bar{c}$.) The sequence (3) clearly satisfies the hypothesis of Lemma 4.1, therefore $D_{1}=D_{2}^{*}$. Thus (i) holds in this case by taking $D_{1}$ as the required $D$.

Suppose $n=4$. Then $[a, b]$ and $[c, d]$ are connected by a strongly normal five-termed sequence of transposes of one of the two following forms

$$
\begin{aligned}
& {[\bar{a}, \bar{b}]=\left[a_{0}, b_{0}\right] \nearrow\left[a_{1}, b_{1}\right] \searrow\left[a_{2}, b_{2}\right] \nearrow\left[a_{3}, b_{3}\right] \searrow\left[a_{4}, b_{4}\right]=[\bar{c}, \bar{d}]} \\
& {[\bar{a}, \bar{b}]=\left[a_{0}, b_{0}\right] \searrow\left[a_{1}, b_{1}\right] \nearrow\left[a_{2}, b_{2}\right] \searrow\left[a_{3}, b_{3}\right] \nearrow\left[a_{4}, b_{4}\right]=[\bar{c}, \bar{d}]}
\end{aligned}
$$

with the associated diamonds satisfying the conclusion of Theorem 3.1. By duality we need only consider the case when (4) holds. Then the associated diamonds satisfy one of the two following conditions:

$$
\begin{aligned}
& D_{1} \underset{(1)}{\longrightarrow} D_{2}^{*} \text { and } D_{2} \underset{(2)}{\longrightarrow} D_{3}, \\
& D_{1} D_{2} \text { and } D_{1}=D_{2}^{*} .
\end{aligned}
$$


Since $b_{0} \leqq \bar{c} \leqq a_{3}$, Lemma 4.1 can be applied to the first three transpositions in (4) to infer that $D_{1}$ cannot translate down onto $D_{2}$, thus ruling out (7) and showing that (6) must hold. A second application of Lemma 4.1 yields $D_{1}=D_{2}^{*}$. Thus $[\bar{a}, \bar{b}] /\left[v_{1}, x_{1}\right]=\left[v_{2}, z_{2}\right]$. Since $\bar{c}+v_{2}=a_{4}+a_{2}=v_{3}$, it is clear that the conclusion (ii) of the theorem holds in this case by taking $D_{1}^{\prime}=D_{2}$ and $D_{2}^{\prime}=D_{3}$.

Finally suppose $n=5$. Then $[\bar{a}, \bar{b}]$ and $[\bar{c}, \bar{d}]$ are connected by a strongly normal six-termed sequence

(8) $[\bar{a}, \bar{b}]=\left[a_{0}, b_{0}\right] /\left[a_{1}, b_{1}\right] \searrow\left[a_{2}, b_{2}\right] /\left[a_{3}, b_{3}\right] \searrow\left[a_{4}, b_{4}\right] /\left[a_{5}, b_{5}\right]=[\bar{c}, \bar{d}]$, with the associated diamonds satisfying the condition

$$
D_{1} \underset{(1)}{\longrightarrow} D_{2}^{*}, D_{2} \underset{(2)}{\longrightarrow} D_{3}, D_{3}=D_{4}^{*}
$$

or dually,

$$
[\bar{a}, \bar{b}]=\left[a_{0}, b_{0}\right] \searrow\left[a_{1}, b_{1}\right] /\left[a_{2}, b_{2}\right] \searrow\left[a_{3}, b_{3}\right] /\left[a_{4}, b_{4}\right] \searrow\left[a_{5}, b_{5}\right]=[\bar{c}, \bar{d}]
$$

with the associated diamonds satisfying the condition

$$
D_{1} \underset{(1)}{\longrightarrow} D_{2}^{*}, D_{2} \underset{(2)}{\longrightarrow} D_{3}, D_{3}=D_{4}^{*} \text {. }
$$

(The alternative to (9),

$$
D_{1} \underset{\text { (1) }}{>} D_{2}, D_{2}=D_{3}^{*}, D_{3} \underset{\text { (2) }}{\longrightarrow} D_{4}
$$

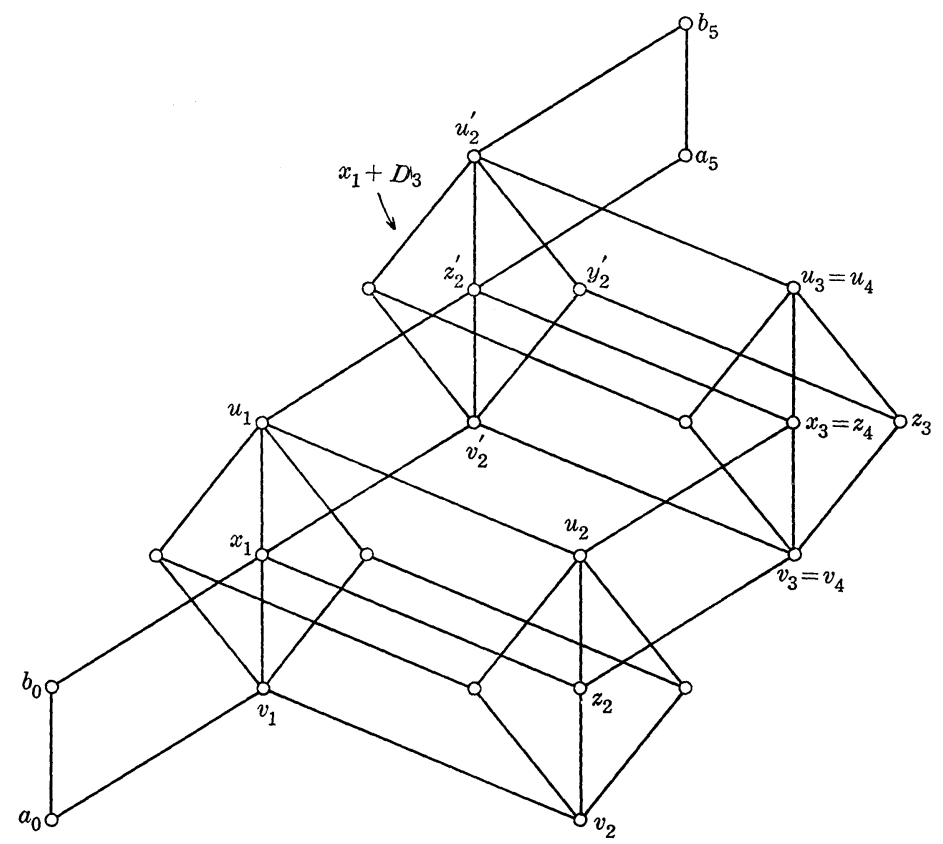

Figure 8 
and the corresponding alternative to (11) can be ruled out by the same argument that showed that (1) leads to a contradiction.) Actually (10) and (11) cannot hold, for then $b_{1} \leqq \bar{b} \leqq \bar{c} \leqq a_{4}$, so that Lemma 4.1 can be applied to the three middle transpositions in (10), showing that the middle formula in (11) fails. Thus we may assume that (8) and (9) hold. Our reasoning is motivated by Fig. 8. Since $u_{1} u_{3}=u_{2}$, we apply the dual of the Transposition Lemma to $D_{3}$ and the sequence $\left[v_{3}, x_{3}\right] \searrow$ $\left[z_{2}, u_{2}\right] \nearrow\left[x_{1}, u_{1}\right]$ to obtain a diamond $x_{1}+D_{3}$. Define the diamond $D_{2}^{\prime}=$ $\left[u_{2}^{\prime}>x_{2}^{\prime}, y_{2}^{\prime}, z_{2}^{\prime}>v_{2}^{\prime}\right]$ so that $\left(D_{2}^{\prime}\right)^{*}=x_{1}+D_{3}$. Using the hypothesis that $a_{5} \geqq b_{0}, u_{2}$ and hence $a_{5} \geqq b_{0}+u_{2}=u_{1}$, one can easily check that

$$
x_{3} \leqq z_{2}^{\prime} \leqq a_{5}, u_{3} \leqq u_{2}^{\prime} \leqq b_{5} \text {. }
$$

Since $\left[x_{3}, u_{3}\right] \nearrow\left[z_{2}^{\prime}, u_{2}^{\prime}\right]$ and $\left[a_{5}, b_{5}\right]$, it follows from (12) that $\left[z_{2}^{\prime}, u_{2}^{\prime}\right] \nearrow$ $\left[a_{5}, b_{5}\right]$. The conclusion (iv) of the theorem clearly holds if we take the above $D_{1}$ and $D_{2}^{\prime}$ as the required $D_{1}^{\prime}$ and $D_{2}^{\prime}$ respectively.

This completes the proof of the Lemma.

Lemma 4.3. Assume that $A_{1} \notin \boldsymbol{H S}(L)$ and $D=[u>x, y, z>v]$ is a diamond in $L$ with $[x, u]$ transposing down onto an interval $[a, b]$. Then either

(i) $a+v=x$ or else

(ii) there exists an element $x^{\prime}$ with $x \leqq x^{\prime}<u$ and an element $b^{\prime}$ with $b \leqq b^{\prime}<u$ such that the nondegenerate diamond $(D)_{x^{\prime}}$ has $x^{\prime}+b^{\prime}$ as its greatest element and $b^{\prime} x^{\prime}$ as its smallest element.

Proof. Suppose (i) fails to hold, or equivalently $a+v<2$. We claim that (ii) holds. In fact, if $a+v=v$ then (ii) trivially holds by taking $x^{\prime}=x$ and $b^{\prime}=b+v$. We can therefore assume that

$$
v<a+v<x \text {. }
$$

Let $b^{\prime}=b+v$, and consider the diamonds $(D)_{a+v}$ and $\overline{(D)}_{a+v}$. By (1), they are nondegenerate. Suppose $u_{1}$ is the greatest element of $(D)_{a+v}$. It follows from the definitions of $(D)_{a+v}$ and $(\bar{D})_{a+v}$ that $\left[a+v, u_{1}\right]$ is one upper edge of $(D)_{a+v}$ (Fig. 9), and furthermore

$$
\left[a+v, u_{1}\right] \supset\left[x, u_{1}+x\right] \text {. }
$$

Let $t=b^{\prime}\left(u_{1}+x\right)$. Then by the transposition of $[x, u]$ onto $[a, b]$, we have

$$
[a+v, t] \longrightarrow\left[x, u_{1}+x\right] .
$$

Consider the three elements $x, u_{1}$, and $t$. If the diamond $\operatorname{ker}\left(x, u_{1}, t\right)$ is nondegenerate, then one of its upper edges transposes up onto 


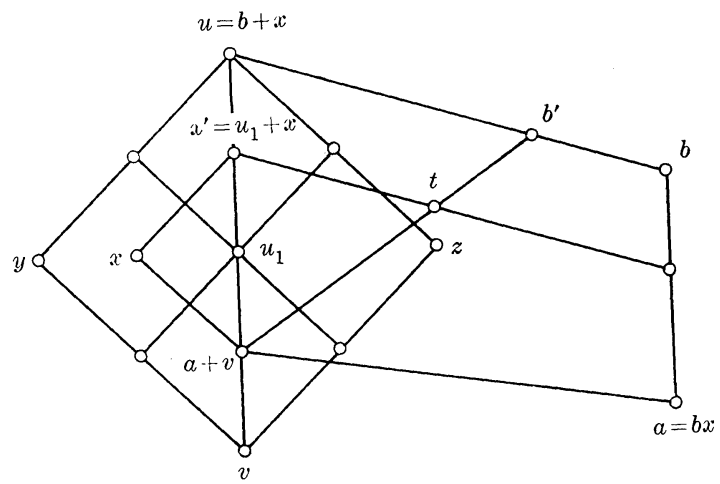

FIGURE 9

a nontrivial subinterval of $\left[u_{1}, u_{1}+x\right]$ and the adjacent edge down onto a nontrivial subinterval of $\left[a+v, u_{1}\right]$ (by an observation in 2.D). Thus $\operatorname{ker}\left(x, u_{1}, t\right)$ translates up and down onto two nondegenerate diamonds by two adjacent edges (by 2.C). It therefore follows that $A_{1} \in \boldsymbol{H} \boldsymbol{S}(L)$, contradicting our hypothesis. Thus the sublattice generated by $\left\{x, u_{1}, t\right\}$ must be distributive. By (2) and (3), $u_{1}$ and $t$ have the same relative complement $x$; hence $u_{1}=t$. Then the elements $b^{\prime}$ and $x^{\prime}=u_{1}+x$ clearly satisfy the requirements in (ii). The lemma is therefore proved.

We now come to the proof of the Main Lemma.

4.4. Proof of the Main Lemma. We first prove the following statement $(\Delta)$ :

(A) Let $L$ be any modular subdirectly irreducible lattice with $A_{1}, A_{2}$, $A_{3} \notin \boldsymbol{H S}(L)$. If $L$ contains a sublattice $M_{3 n}^{+}(n \geqq 1)$ whose greatest element is less than an element $d$ in $L$, then $L$ contains a sublattice $M_{3 n+1}^{+}$with its greatest element less than or equal to the element $d$.

Suppose the given $M_{3 n}^{+}$is formed by $n$ diamonds $D_{i}=\left[u_{i}>x_{i}, y_{i}, z_{i}>v_{i}\right]$ $i=1,2, \cdots, n$, with

$$
\begin{gathered}
{\left[x_{i}, u_{i}\right] \supset\left[v_{i+1}, z_{i+1}\right] \quad(1 \leqq i \leqq n-1)} \\
u_{n}<d .
\end{gathered}
$$

It is well known that since $L$ is a subdirectly irreducible modular lattice there are some nontrivial subintervals $[\bar{a}, \bar{b}]$ of $\left[x_{n}, u_{n}\right]$ and $[\bar{c}, \bar{d}]$ of $\left[u_{n}, d\right]$ that are projective to each other. Therefore by Lemma 4.2 we can choose them so that one of the four conclusions (i) - (iv) of that lemma holds. If (i) holds, then there exists a nondegenerate diamond 
such that one of its lower edges transposes down onto $[\bar{a}, \bar{b}]$ and the adjacent upper edge up onto $[\bar{c}, \bar{d}]$. We can therefore take the sublattice formed by that diamond and $\left(M_{3^{n}}^{+}\right)_{[\bar{a}, \bar{b}]}$ as the required $M_{3^{n+1}+1}^{+}$. If (ii) or (iv) holds, then $[\bar{a}, \bar{b}]$ translates up onto the first stage of a sublattice of the form $M_{3^{2}}^{+}$and this sublattice forms with $\left(D_{n}\right)_{[\bar{a}, \bar{b}]}$ a sublattice having $A_{1}$ as a homomorphic image. We have a contradiction. Therefore it remains to consider the case when (iii) holds. Then we have two diamonds $D_{i}^{\prime}=\left[u_{i}^{\prime}>x_{i}^{\prime}, y_{i}^{\prime}, z_{i}^{\prime}>v_{i}^{\prime}\right], i=1,2$, such that $D_{1}^{\prime} \nearrow_{(2)} D_{2}^{\prime}$ and

$$
\begin{gathered}
{[\bar{a}, \bar{b}] \supset\left[\bar{x}_{1}^{\prime}, \bar{u}_{1}^{\prime}\right],[\bar{c}, \bar{d}] \longrightarrow\left[x_{2}^{\prime}, u_{2}^{\prime}\right],} \\
\bar{b} u_{2}^{\prime}=u_{1}^{\prime} .
\end{gathered}
$$

By substituting $\left(M_{3^{n}}^{+}\right)_{[\bar{a}, \bar{b}]}$ for $M_{3^{n}}^{+}$, we can assume that

$$
[\bar{a}, \bar{b}] \succ\left[x_{n}, u_{n}\right] \text {. }
$$

Then by substituting $\left[\left(u_{n}+u_{1}^{\prime}\right) \bar{a}, u_{n}+u_{1}^{\prime}\right]$ for $[\bar{a}, \bar{b}]$, we can furthermore assume that

$$
u_{1}^{\prime}+u_{n}=\bar{b} .
$$

We are in the situation as illustrated in Fig. 10.

Since many diamonds will be involved, therefore for the sake of simplicity we tacitly assume that the vertices of a diamond written $D_{i}^{\prime}$ will be in symbols $\left[u_{i}^{\prime}>x_{i}^{\prime}, y_{i}^{\prime}, z_{i}^{\prime}>v_{i}^{\prime}\right]$. Suppose there exists an element $t$ such that either the sublattice generated by $\left\{t, u_{n}, \bar{a}\right\}$ or the one generated by $\left\{t, u_{1}^{\prime}, \bar{a}\right\}$ is not distributive. Then either $\operatorname{ker}\left(t, u_{n}, \bar{a}\right)$ or $\operatorname{ker}\left(t, u_{1}^{\prime}, \bar{a}\right)$ is nondegenerate. If $\operatorname{ker}\left(t, u_{n}, \bar{a}\right)$ is nondegenerate, then it translates up onto a subinterval $\alpha$ of $[\bar{a}, \bar{b}]$ and down onto a subinterval $\beta$ of $\left[x_{n}, x_{n}\right]$, and since $\beta$ must be nontrivial we can take the sublattice formed by this diamond and $\left(M_{3^{n}}^{+}\right)_{\beta}$ as the required $M_{3^{n+1}}^{+}$. So assume that $\operatorname{ker}\left(t, u_{1}^{\prime}, \bar{a}\right)=D_{3}^{\prime}$ is non-degenerate with $u_{3}^{\prime}$ as its greatest element. Then it translates up onto a subinterval $\left[m, m^{\prime}\right]$ of $[a, b]$ and down onto a subinterval $\left[s_{1}, s_{1}^{\prime}\right]$ of $\left[x_{1}^{\prime}, u_{1}^{\prime}\right]$. Furthermore $\left[s_{1}, s_{1}^{\prime}\right]$ is the image of $\left[m, m^{\prime}\right]$ under the transposition $[\bar{a}, \bar{b}]$ onto $\left[x_{1}, u_{1}^{\prime}\right]$. Then using mainly the fact that $\bar{b} u_{2}^{\prime}=u_{1}^{\prime}$, one can check that $D_{3}^{\prime}$ generates with $\left(D_{1}^{\prime} \cup D_{2}^{\prime}\right)_{\left.\iota_{1}, s_{1}^{\prime}\right]}$ a sublattice which has $A_{2}$ as a homomorphic image (see Fig. 11 for motivation), contradicting the hypothesis that $A_{2} \notin$ $\boldsymbol{H S}(L)$.

We can therefore assume that the four lattices generated by $\left\{y_{n}\right.$, $\left.u_{1}^{\prime}, \bar{a}\right\},\left\{z_{n}, u_{1}^{\prime}, \bar{a}\right\},\left\{y_{1}^{\prime}, u_{n}, \bar{a}\right\},\left\{z_{1}^{\prime}, u_{n}, \bar{a}\right\}$ respectively are distributive. We infer that

$$
\bar{a} y_{n}+\bar{a} u_{1}^{\prime}=\bar{a}\left(y_{n}+u_{1}^{\prime}\right) \text { and } \bar{a} z_{n}+\bar{a} u_{1}^{\prime}=\bar{a}\left(z_{n}+u_{1}^{\prime}\right),
$$




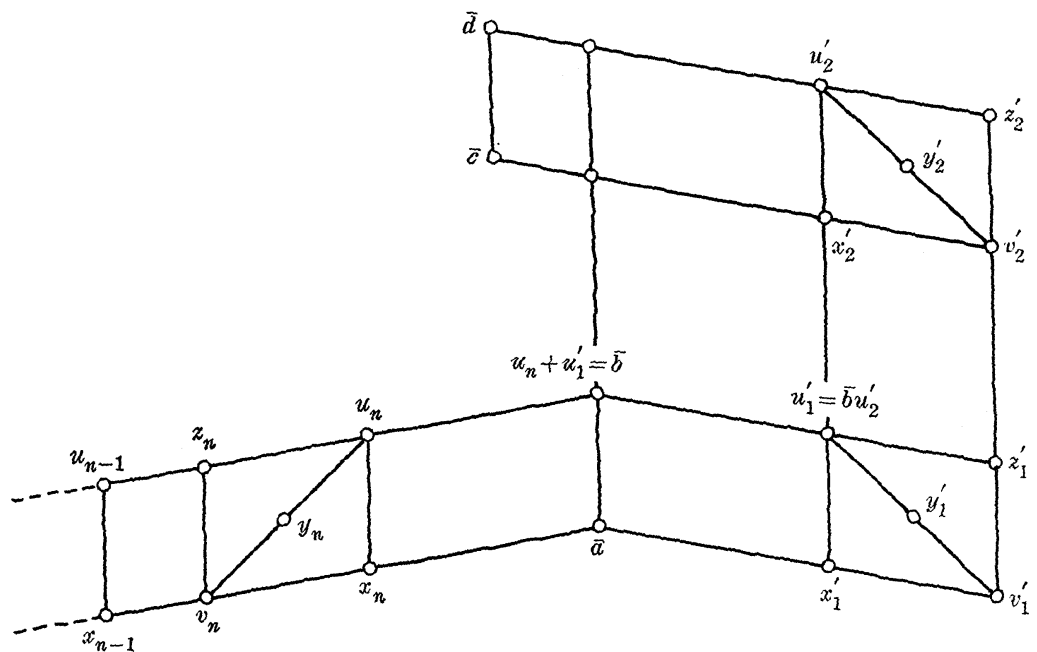

FIgURE 10

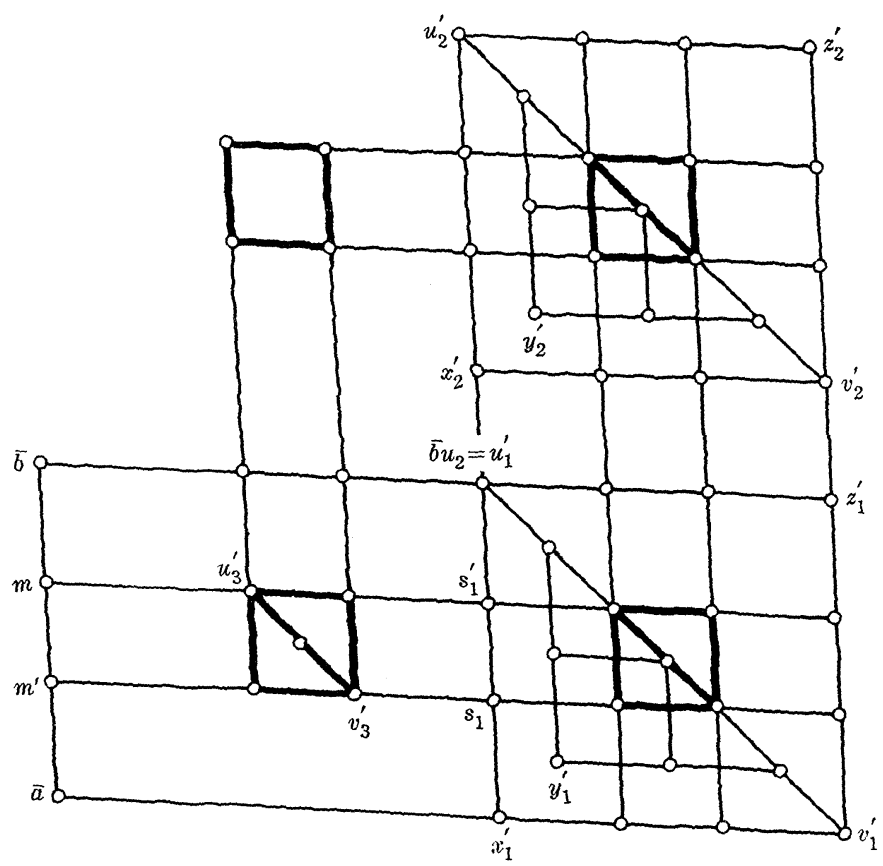

FIGURE 11

(8)

$$
\bar{a} y_{1}^{\prime}+\bar{a} u_{n}=\bar{a}\left(y_{1}^{\prime}+u_{n}\right) \text { and } \bar{a} z_{1}^{\prime}+\bar{a} u_{n}=\bar{a}\left(z_{1}^{\prime}+u_{n}\right) \text {. }
$$

By adding the two equalities in (7), we obtain

$$
\begin{aligned}
v_{n}+x_{1}^{\prime} & =\bar{a}\left(y_{n}+u_{1}^{\prime}\right)+\bar{a}\left(z_{n}+u_{1}^{\prime}\right)=\bar{a}\left(y_{n}+u_{1}^{\prime}+\bar{a}\left(z_{n}+u_{1}^{\prime}\right)\right) \\
& =\bar{a}\left(y_{n}+\left(u_{1}^{\prime}+\bar{a}\right)\left(z_{n}+u_{1}^{\prime}\right)\right)=\bar{a}\left(y_{n}+z_{n}+u_{1}^{\prime}\right) \\
& =\bar{a}\left(u_{n}+u_{1}^{\prime}\right)=\bar{a} .
\end{aligned}
$$


The last equality is due to (6). Similarly, by adding side by side the two equalities in (8) we obtain

$$
v_{1}^{\prime}+x_{n}=\bar{a} .
$$

By (3), (5) and (9), the Transposition Lemma applies to $D_{n}$, yielding a diamond $D_{6}^{\prime}=u_{1}^{\prime} D_{n}$ with

$$
\left[x_{1}^{\prime}, u_{1}^{\prime}\right] \succ_{\perp}\left[x_{6}^{\prime}, u_{6}^{\prime}\right] \text { and } D_{n} \underset{(1)}{\supset} D_{6}^{\prime} \text {. }
$$

Furthermore, by (10) we have

$$
v_{1}^{\prime}+x_{6}^{\prime}=v_{1}^{\prime}+u_{1}^{\prime} x_{n}=u_{1}^{\prime}\left(v_{1}^{\prime}+x_{n}\right)=u_{1}^{\prime} \bar{a}=x_{1}^{\prime} .
$$

By (11) and (12) the Transposition Lemma applies to $D_{1}^{\prime}$, yielding a diamond $D_{7}^{\prime}$ with $D_{7}^{\prime}=u_{6}^{\prime} D_{1}^{\prime}$ and

$$
\left[x_{7}^{\prime}, u_{7}^{\prime}\right]=\left[x_{6}^{\prime}, u_{6}^{\prime}\right] \text { and } D_{1}^{\prime} \underset{(1)}{>} D_{7}^{\prime} \text {. }
$$

If $v_{7}^{\prime}+v_{6}^{\prime}=x_{6}^{\prime}$, then the Transposition Lemma applies to $D_{6}^{\prime}$, yielding a sublattice $D_{8}^{\prime}=z_{7}^{\prime} D_{6}$ with $\left[v_{7}^{\prime}, z_{7}^{\prime}\right]$ as one of its upper edges. Then the sublattice formed by $D_{2}^{\prime}, D_{7}^{\prime}, D_{8}^{\prime}$ has $A_{1}$ as a homomorphic image, contradicting the hypothesis that $A_{1} \notin \boldsymbol{H S}(L)$. Therefore the conclusion (ii) of Lemma 4.3 applied to the diamond $D_{6}^{\prime}$ and the interval $\left[v_{7}^{\prime}, z_{7}^{\prime}\right]$ must hold. We have therefore an element $e$ and an element $x_{9}^{\prime}$ with

$$
x_{6}^{\prime} \leqq x_{9}^{\prime}<u_{6}^{\prime} \text { and } z_{7}^{\prime} \leqq e<u_{6}^{\prime},
$$

such that if $D_{9}^{\prime}=\left(D_{6}^{\prime}\right)_{x_{9}^{\prime}}$, then

$$
e+x_{9}^{\prime}=u_{9}^{\prime}=u_{6}^{\prime} \text { and } e x_{9}^{\prime}=v_{9}^{\prime} \text { (Fig. 12) . }
$$

The diamond $D_{9}^{\prime}$ and the element $e$ are thus elements of the interval $\left[v_{6}^{\prime}, u_{6}^{\prime}\right]$. By the transposition of $\left[v_{6}^{\prime}, u_{6}^{\prime}\right]$ onto $\left[v_{n}, u_{n}\right], x_{9}^{\prime}$ and $e$ are mapped respectively into $x_{10}^{\prime}=v_{n}+x_{9}^{\prime}$ and $f=v_{n}+e$, so that $x_{n} \leqq$ $x_{10}^{\prime}<u_{10}^{\prime}$ and if $D_{10}^{\prime}=\left(D_{n}\right)_{x_{10}^{\prime}}$, then

$$
\begin{gathered}
D_{9}^{\prime} \underset{(1)}{\longrightarrow} D_{10}^{\prime}, \\
{\left[e, u_{6}^{\prime}\right]=\left[e, u_{9}^{\prime}\right] \longrightarrow\left[f, u_{10}^{\prime}\right]=\left[f, u_{n}\right]} \\
f+x_{10}^{\prime}=u_{10}^{\prime} \text { and } f x_{10}^{\prime}=v_{10}^{\prime} .
\end{gathered}
$$

We have used here (15) and the fact that $\left(D_{6}^{\prime}\right)_{x_{9}^{\prime}}=D_{9}^{\prime}$. Let

$$
g=e+z_{2}^{\prime} \text { and } D_{11}^{\prime}=\left(D_{2}^{\prime}\right)_{g}\left(u_{11}^{\prime}=u_{2}^{\prime}\right),
$$

then $\left[e, u_{6}^{\prime}\right]$ transposes up onto $\left[v_{11}^{\prime}, x_{11}^{\prime}\right]$. With (17) and

$$
u_{2}^{\prime} u_{n}=u_{2}^{\prime}\left(\bar{b} u_{n}\right)=u_{1}^{\prime} u_{n}=u_{6}^{\prime},
$$




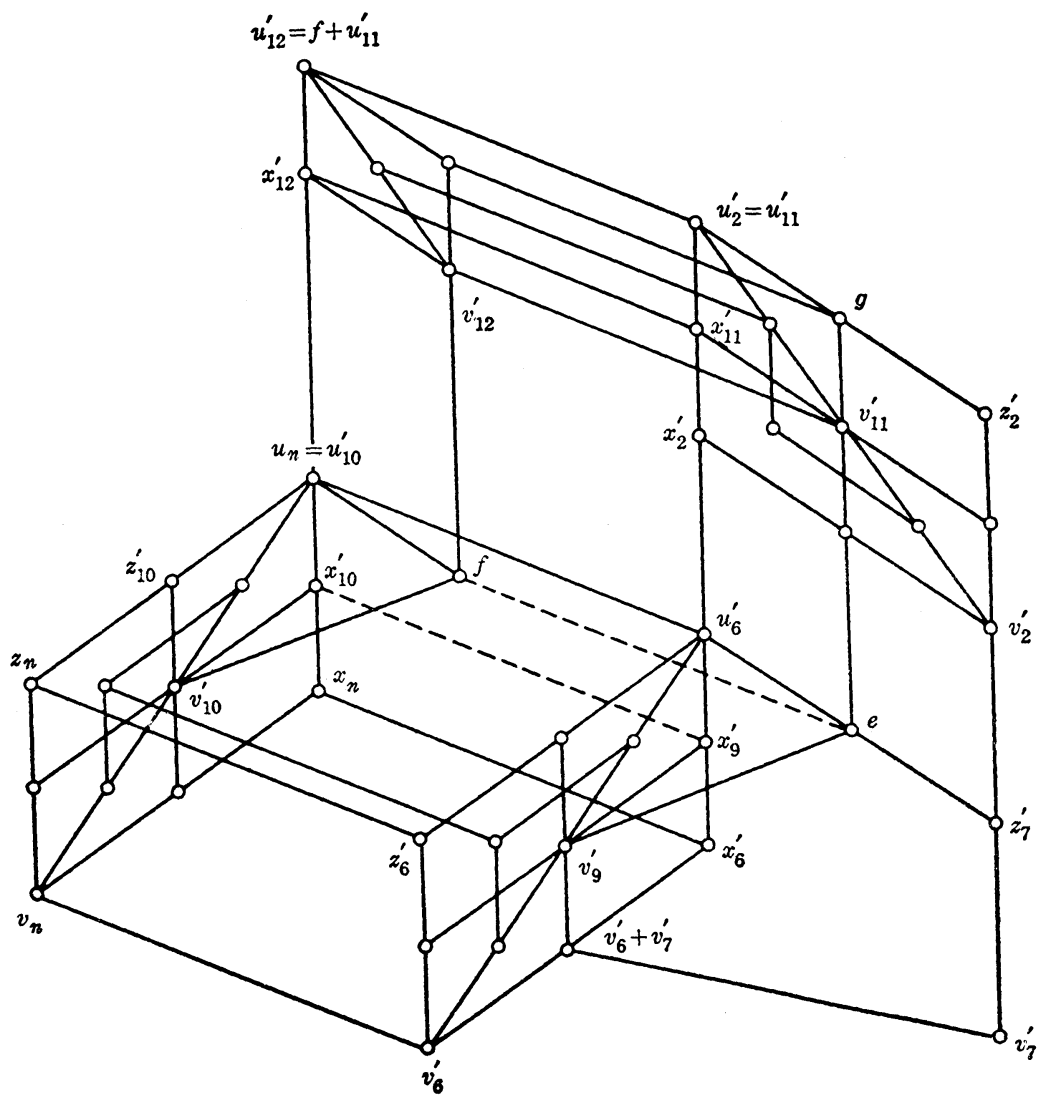

FIGURE 12

the dual of Transposition Lemma applies to $D_{11}^{\prime}$, yielding a diamond $D_{12}^{\prime}=f+D_{11}^{\prime}$ so that $\left[f, u_{n}\right]$ transposes up onto $\left[v_{12}^{\prime}, x_{12}^{\prime}\right]$ and

$$
u_{12}^{\prime}=f+u_{11}^{\prime} \leqq u_{n}+u_{2}^{\prime} \leqq \bar{d} \leqq d .
$$

We now consider the configuration formed by $f, D_{10}^{\prime}, D_{12}^{\prime}$ and $\bigcup_{i=1}^{n-1} D_{i}$. If the sublattice generated by $\left\{f, x_{10}^{\prime}, z_{10}^{\prime}\right\}$ is distributive, then $z_{10}^{\prime}$ and $f$ are equal, since by (18) they have the same relative complement $x_{10}^{\prime}$ in this lattice. Thus we have

$$
\left[z_{10}^{\prime}, u_{10}^{\prime}\right] \longrightarrow\left[v_{12}^{\prime}, x_{12}^{\prime}\right] .
$$

Furthermore, $\left[v_{10}^{\prime}, z_{10}^{\prime}\right]$ transposes down onto a subinterval of $\left[v_{n}, z_{n}\right]$, hence onto a subinterval of $\left[x_{n-1}, x_{n-1}\right]$ if $n \geqq 2$. Therefore $\left[v_{10}^{\prime}, z_{10}^{\prime}\right]$ translates down onto a nontrivial homothetic image of $D_{n-1}$ if $n \geqq 2$. Using (22), we have either a contradiction to the hypothesis that $A_{1} \notin \boldsymbol{H S}(L)$ (if $n \geqq 2$ ) or else the conclusion of $(4)$ holds (when $n=1$ ). So we can assume that the sublattice generated by $\left\{f, x_{10}^{\prime}, z_{10}^{\prime}\right\}$ is nondistributive. Then $\operatorname{ker}\left(f, x_{10}^{\prime}, z_{10}^{\prime}\right)$ is nondegenerate diamond which 
translates up onto a subinterval of $\left[f, u_{n}\right]$ and down onto a subinterval of $\left[v_{10}^{\prime}, z_{10}^{\prime}\right]$ by two opposite edges. Therefore, $\operatorname{ker}\left(f, x_{10}^{\prime}, z_{10}^{\prime}\right)$ translates up onto a subinterval $\gamma$ of $\left[v_{12}^{\prime}, x_{12}^{\prime}\right]$ and down onto a subinterval $\delta$ of $\left[x_{n-1}, u_{n-1}\right]$ by two opposite edges, and the two intervals $\gamma$ and $\delta$ are nontrivial. Using the fact that $u_{12}^{\prime} \leqq d$ in (21), we can therefore take the sublattice formed by $\operatorname{ker}\left(f, x_{10}^{\prime}, z_{10}^{\prime}\right),\left(D_{12}^{\prime}\right)_{r}$ and $\left(\bigcup_{i=1}^{n-1} D_{i}\right)_{\delta}$ as the required $M_{3 n+1}^{+}$. This completes the proof of the statement $(\Delta)$.

We will complete the proof of the lemma by showing that if the lattice $L$ given in the lemma is infinite dimensional, then $L$ contains a sublattice $M_{3}^{+}$for every positive integer $k$, and if $L$ is of dimension $k+1(\geqq 2)$, then $L$ contains a sublattice $M_{3}$. Observe that in either case there exists a nondegenerate diamond $D=[u>x, y, z>v]$ in $L$.

Case (i). $L$ is infinite dimensional. Then for every $k$, there exists a sequence $d_{1}<d_{2}<\cdots<d_{k-1}$ in $L$ with either $u<d_{1}$ or $d_{n-1}<v$, or $x<d_{1}<d_{2} \cdots<d_{k-1}<u$. In the first two cases we can apply the statement (1) (or its dual) $k-1$ successive times with $d$ replaced successively by $d_{1}, d_{2}, \cdots, d_{k-1}$ (or $\left.d_{k-1}, d_{k-2}, \cdots, d_{1}\right)$ in order to construct the required $M_{3}^{+}{ }_{k}$. In the last case, by replacing $D$ by $\overline{(D)}_{d_{1}}$ we go back to the first.

Case (ii). $L$ is finite dimensional. Then we can assume that the edges of $D$ are prime quotients and the term $v$ of $D$ is a minimal element of all smallest elements of nondegenerate diamonds in $L$. By the dual of the statement $(\Delta), v$ must be the smallest element of $L$. Then since $\operatorname{dim}(L)=k+1$, we can choose a sequence $d_{1}<d_{2}<\cdots<$ $d_{k-1}$ with $d_{1}>u_{1}$. As in Case (i), we now apply the statement ( $(4)$ $k-1$ successive times with $d$ replaced successively by $d_{1}, d_{2}, \cdots, d_{k-1}$ in order to construct a sublattice $M_{3}^{+}$which is the required sublattice $M_{3} k$ since $\operatorname{dim}(L)=k+1$.

This completes the proof of the lemma.

Added in proof. Using different techniques, K. Baker independently announced without proof that the variety $M_{3}^{\infty}$ is finitely based in [2].

\section{REFERENCES}

1. K. A. Baker, Equational classes of modular lattices, Pacific J. Math., 28 (1969), 915.

2. - Equational axioms for classes of lattices, Bull. Amer. Math. Soc., 77 (1971), 97-102.

3. G. Birkhoff, Lattice Theory, Amer. Math. Soc. Colloq. Publ. 25, Providence, 1967.

4. G. Grätzer, Equational classes of lattices, Duke Math. J., 33 (1966), 613-622.

5. D. X. Hong, Covering Relations among Lattice Varieties Thesis, Vanderbilt U., 1970, (available from University Microfilms, P. O. Box 1764, Ann Arbor, Mich.) 
6. B. Jónsson, Arguesian lattices of dimension $n \leqq 4$, Math. Scand., 7 (1959), 133-145. 7. - Algebras whose congruence lattices are distributive, Math. Scand., 21 (1967), 110-121.

8. — Equational classes of lattices, Math. Scand., 22 (1968), 187-196.

9. R. McKenzie, Equational bases and non-modular lattice Varieties, To appear in Trans. Amer. Math. Soc.

10. - On equational theories of lattices, Math. Scand., 24 (1970).

11. R. Wille, Primitive Lange und primitive weite bei modularen verbanden, Math. Z., 108 (1969), 129-136.

Received November 25, 1970 and in revised form October 29, 1971. These results are taken from the author's doctoral thesis, supervised by Professor Bjarni Jónsson at Vanderbilt University. This work was supported by NSF Grants GP-11804 and RG-1452.

UNIVERSITY OF SAIGON 



\section{PACIFIC JOURNAL OF MATHEMATICS}

\section{EDITORS}

\section{H. SAMELSON}

Stanford University

Stanford, California 94305

\section{R. HOBBY}

University of Washington Seattle, Washington 98105

\section{J. DugundJI}

Department of Mathematics University of Southern California Los Angeles, California 90007

RICHARD ARENS

University of California Los Angeles, California 90024

\section{ASSOCIATE EDITORS}
E. F. BECKENBACH
B. H. Neumann
F. WoLF
K. YoSHIDA

\section{SUPPORTING INSTITUTIONS}

UNIVERSITY OF BRITISH COLUMBIA CALIFORNIA INSTITUTE OF TECHNOLOGY

UNIVERSITY OF CALIFORNIA

MONTANA STATE UNIVERSITY

UNIVERSITY OF NEVADA

NEW MEXICO STATE UNIVERSITY

OREGON STATE UNIVERSITY

UNIVERSITY OF OREGON

OSAKA UNIVERSITY

\author{
UNIVERSITY OF SOUTHERN CALIFORNIA \\ STANFORD UNIVERSITY \\ UNIVERSITY OF TOKYO \\ UNIVERSITY OF UTAH \\ WASHINGTON STATE UNIVERSITY \\ UNIVERSITY OF WASHINGTON \\ * * * * \\ AMERICAN MATHEMATICAL SOCIETY \\ NAVAL WEAPONS CENTER
}

The Supporting Institutions listed above contribute to the cost of publication of this Journal, but they are not owners or publishers and have no responsibility for its content or policies.

Mathematical papers intended for publication in the Pacific Journal of Mathematics should be in typed form or offset-reproduced, (not dittoed), double spaced with large margins. Underline Greek letters in red, German in green, and script in blue. The first paragraph or two must be capable of being used separately as a synopsis of the entire paper. The editorial "we" must not be used in the synopsis, and items of the bibliography should not be cited there unless absolutely necessary, in which case they must be identified by author and Journal, rather than by item number. Manuscripts, in duplicate if possible, may be sent to any one of the four editors. Please classify according to the scheme of Math. Rev. Index. to Vol. 39. All other communications to the editors should be addressed to the managing editor, Richard Arens, University of California, Los Angeles, California, 90024.

50 reprints are provided free for each article; additional copies may be obtained at cost in multiples of 50 .

The Pacific Journal of Mathematics is published monthly. Effective with Volume 16 the price per volume (3 numbers) is $\$ 8.00$; single issues, $\$ 3.00$. Special price for current issues to individual faculty members of supporting institutions and to individual members of the American Mathematical Society: $\$ 4.00$ per volume; single issues $\$ 1.50$. Back numbers are available.

Subscriptions, orders for back numbers, and changes of address should be sent to Pacific Journal of Mathematics, 103 Highland Boulevard, Berkeley, California, 94708.

PUBLISHED BY PACIFIC JOURNAL OF MATHEMATICS, A NON-PROFIT CORPORATION

Printed at Kokusai Bunken Insatsusha (International Academic Printing Co., Ltd.), 270, 3-chome Totsuka-cho, Shinjuku-ku, Tokyo 160, Japan. 


\section{Pacific Journal of Mathematics}

\section{Vol. 40, No. $3 \quad$ November, 1972}

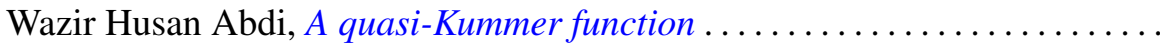

Vasily Cateforis, Minimal injective cogenerators for the class of modules of

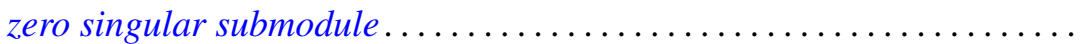

W. Wistar (William) Comfort and Anthony Wood Hager, Cardinality of

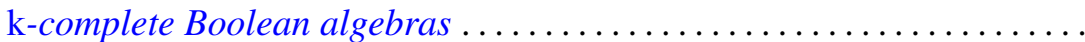

Richard Brian Darst and Gene Allen DeBoth, Norm convergence of martingales of Radon-Nikodym derivatives given a $\sigma$-lattice ..........

M. Edelstein and Anthony Charles Thompson, Some results on nearest points and support properties of convex sets in $c_{0} \ldots \ldots \ldots \ldots \ldots$

Richard Goodrick, Two bridge knots are alternating knots .

Jean-Pierre Gossez and Enrique José Lami Dozo, Some geometric properties related to the fixed point theory for nonexpansive mappings ..........

Dang Xuan Hong, Covering relations among lattice varieties .............

Carl Groos Jockusch, Jr. and Robert Irving Soare, Degrees of members of $\Pi_{1}^{0}$

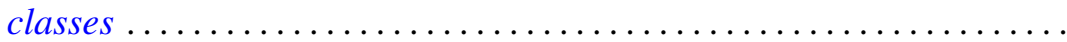

565

575

605

Leroy Milton Kelly and R. Rottenberg, Simple points in pseudoline

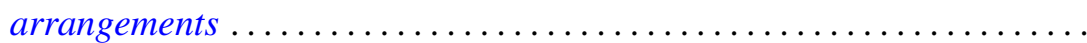

Joe Eckley Kirk, Jr., The uniformizing function for a class of Riemann surfaces....

Glenn Richard Luecke, Operators satisfying condition $\left(G_{1}\right)$ locally ... 629

T. S. Motzkin, On L $(S)$-tuples and l-pairs of matrices ... . .

Charles Estep Murley, The classification of certain classes of torsion free

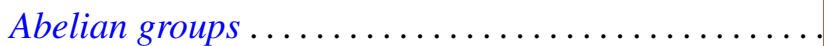

Louis D. Nel, Lattices of lower semi-continuous functions and associated topological spaces.

David Emroy Penney, II, Establishing isomorphism between tame prime

knots in $E^{3}$. . .

Daniel Rider, Functions which operate on $\mathscr{F} L_{p}(T), 1<p<2$

Thomas Stephen Shores, Injective modules over duo rings ...

Stephen Simons, A convergence theorem with boundary. .

703

Stephen Simons, Maximinimax, minimax, and antiminimax theorems and a

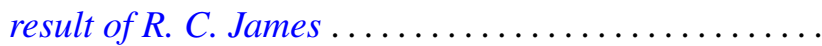

Stephen Simons, On Ptak's combinatorial lemma ........

Stuart A. Steinberg, Finitely-valued $f$-modules............

Pui-kei Wong, Integral inequalities of Wirtinger-type and fourth-order

elliptic differential inequalities .

Yen-Yi Wu, Completions of Boolean algebras with partially additive

operators ..................................

Phillip Lee Zenor, On spaces with regular $G_{\delta}$-diagonals . . . 